\title{
A Limiting Channel Capacity of Visual Perception: Spreading Attention Divides the Rates of Perceptual Processes
}

\author{
Joseph S. Lappin ${ }^{1,2} \cdot$ Adriane E. Seiffert $^{1} \cdot$ Herbert H. Bell ${ }^{2}$ \\ Published online: 21 February 2020 \\ (C) The Psychonomic Society, Inc. 2020
}

\begin{abstract}
This study investigated effects of divided attention on the temporal processes of perception. During continuous watch periods, observers responded to sudden changes in the color or direction of any one of a set of moving objects. The set size of moving objects was a primary variable. A simple detection task required responses to any display change, and a selective task required responses to a subset of the changes. Detection rates at successive points in time were measured by response time (RT) hazard functions.

The principal finding was that increasing the set size divided the detection rates - and these divisive effects were essentially constant over time and over the time-varying influence of the target signals and response tasks. The set size, visual target signal, and response task exerted mutually invariant influence on detection rates at given times, indicating independent joint contributions of parallel component processes. The lawful structure of these effects was measured by RT hazard functions but not by RTs as such. The results generalized the time-invariant divisive effects of set size on visual process rates found by Lappin, Morse, \& Seiffert (Attention, Perception, \& Psychophysics, 78, 2469-2493, 2016). These findings suggest that the rate of visual perception has a limiting channel capacity.
\end{abstract}

Keywords Attention: Divided Attention and Inattention - Attention: Theoretical and Computational Models $\cdot$ Reaction time methods $\cdot$ Hazard functions $\cdot$ Process rates $\cdot$ Channel capacity

\section{Introduction}

We know less than meets the eye. Visual processes convert variations in optical energy into information, but potential information is often not consciously perceived. When attention is distributed over multiple objects and events, recognition of any given object or event becomes slower and less accurate. This study examined how distributed attention affected temporal processes for detecting a target change in any one of several moving objects.

Joseph S. Lappin

joe.lappin@vanderbilt.edu

1 Vanderbilt University, Nashville, TN, USA

2 Discerning Technologies LLC, San Diego, CA, USA
Objects and events that are entirely visible, centered in the field of view, and seemingly salient are surprisingly vulnerable to the limitations of conscious perceptual awareness. ${ }^{1}$ Indeed, failures of perceptual awareness are more common than we realize (Levin, Momen, \& Drivdahl, 2000). These failures have been documented in many different everyday settings (e.g., Chabris \& Simons, 2009; Drew, Vo, \& Wolfe, 2013; Varakin, Levin, \& Fidler, 2004; Wolfe, Soce, \& Schill, 2017; Young \& Regan, 2007). The limitations of awareness are also well documented in controlled experiments (e.g., Cohen, Cavanagh, Chun, \&

\footnotetext{
${ }^{1}$ Awareness is not a well-defined concept. Koenderink (2012, 2018, pp. 23 25 ) offers a comprehensive description of visual awareness, showing that the contents of awareness are inherently subjective. The operational definition in the present study was the observer's detection of a change in an object's color or motion direction. Response times (RTs) were assumed to reflect the speed of conscious detection. The term 'awareness' refers here to a state in which one is conscious of and can respond to a particular object, event, or pattern. The processes by which one becomes aware of particular information are unknown but include sensory, attentional, perceptual, and cognitive processes.
} 
Nakayama, 2012; Dux \& Marois, 2009; Eckstein, Koehler, Welbourne, \& Akbas, 2017; Eriksen \& Johnson, 1964; Kennedy, Rawding, Most, \& Hoffman, 2014; Lappin, Morse, \& Seiffert, 2016; Mack, 2003; Mack \& Rock, 1998; Most, Chun, Widders, \& Zald, 2005; Simons \& Chabris, 1999). Failures of awareness are also recognized as causes of accidents in auto traffic (Pammer, Sabadas, \& Lentern, 2018; Strayer, Drews, \& Johnston, 2003) and in military scenarios (Air Combat Command, 2009). These phenomena have inspired descriptive labels such as "inattentional blindness," "change blindness," "attentional blink," "emotion-induced blindness," and "distraction".

The usual method for evaluating perceptual capacity has been to quantify immediate memory for briefly displayed items that are no longer visible when tested. This "span of apprehension", measured by immediate memory, was once considered a fundamental limiting factor in the real-time process of perception. Wundt (1894) described the basic result: ". . f four, and sometimes even five disconnected impressions (letters, numerals, or lines of different direction) may be distinctly perceived. [p. 243]." Perhaps, however, this limited span derives more from memory than from real-time perception and attention. Essentially the same phenomena are often now attributed to "visual working memory" (VWM).

A vast literature has found that VWM capacity limits of about 3-5 independent objects are (a) robust over many different types of objects and response methods, (b) minimally affected by the number of dimensions of each object, (c) selectable from larger sets of displayed objects, and (d) potentially composed of meaningful patterns, words, or "chunks" (e.g., Averbach \& Coriell, 1961; Eriksen \& Lappin, 1967; Fougnie \& Marois, 2006; Lappin, 1967; Lappin \& Ellis, 1970; Luck \& Vogel, 1997, 2013; Miller, 1956; Pratte, Park, Rademaker, \& Tong, 2017; Sperling, 1960; Vogel, Woodman, \& Luck, 2001; Woodworth \& Schlossberg, 1954; Zhang \& Luck, 2011). An important question, however, is whether this limitation involves only memory for previous observations. Is perceptual capacity also limited prior to working memory?

One hypothesis is that "pre-attentive" vision operates in parallel with unlimited capacity. Capacity limits are often thought to occur at a second stage of selective attention (e.g., Palmer et al., 2011; Treisman \& Gelade, 1980; Wolfe, Cave, \& Franzel, 1989). Research on failures of awareness, cited above, challenges this hypothesis - suggesting instead that perceptual awareness is competitive and selective from the beginning. As will be seen, the present results also suggest that the whole perceptual process may operate with limited capacity from sensory input to behavioral output, with no distinct bottleneck in selective attention. How such capacity limitations of perception blend into those of working memory is not clear, though target detections in the present study were evidently governed mainly by perception at the moment of target occurrence and not by memory of the input information.
A basic problem in evaluating perceptual capacity is that the input information is not objectively quantifiable. Optical input varies with the observer's vantage point; and visual information entails spatial, temporal, and environmental contexts and relevance to the observer's actions, purposes, knowledge, and comprehension. ${ }^{2}$ Input information may be experimentally quantified by constructing patterns from independently varied components. Such information, however, derives from an experimenter's assumptions and choices about the elements of information, rather than from empirical evidence about the effective visual complexity of the stimulus patterns. In the present study, the visual complexity of displayed information was not known beforehand, except for the qualitative assumption that complexity increased with the set size of potential target objects.

This study and a closely related preceding study (Lappin et al., 2016) measured the rate of output information. If the output rate approaches a maximum that is invariant with the amount of input, then that maximum rate may be regarded as a hypothetical limiting 'channel capacity'. ${ }^{3}$

The present study investigated three interrelated basic issues: How do capacity limitations of conscious perception influence temporal processes of perception? Is the capacity of visual perception a limited span of objects or a limited rate of operation? How does perceptual awareness interact with visual processes? Potential hypotheses about these issues will be examined in relation to the obtained results.

Previously, we found that a single capacity parameter $(\sim 25$ bits/s) was consistent with a wide range of performance - over wide variations in distributed attention, visual motion information, and response time (RT) (Lappin et al., 2016). Moreover, the channel capacity of attention and the process of visual motion integration had concurrent independent influence on the rate of detection at any given time. Thus, detection rates, $b^{\prime}(t)$, in bits/s, were well described as products of these two processes specified by four parameters:

$b^{\prime}(t)=M(t) \cdot A^{-1}(n) \cdot C$

where $C \approx 25$ bits/s was the channel capacity; $A(n)=n=$ the number of visually attended objects; and visual motion

\footnotetext{
2 'Information' was also not an objective quantity in Shannon's (1948) mathematical theory. Shannon quantified transmitted information relative to the receiver's prior uncertainty about the potential signals, which were known to both sender and receiver prior to communication.

${ }^{3}$ The concept of 'capacity' refers in this paper to a hypothesized 'channel capacity', measured by target detection rates, in bits/s. The present use is analogous to Shannon's (1948) concept of channel capacity. The present concept of 'capacity' should not be confused with a different definition and measure of capacity developed by James Townsend \& colleagues (e.g., Townsend \& Eidels, 2011). Townsend's measure is a ratio of cumulative hazard functions - the hazard function for detecting two concurrent targets (OR or AND tasks) relative to the prediction for two independent parallel channels, estimated from the hazard functions for the individual targets. The present approach and Townsend's are compatible, but, unfortunately, the term 'capacity' refers to different concepts in the two methodologies.
} 
integration increased proportionally with time over a limited period of integration, $M(t)=(t-c) /(k-c)$ if $c \leq t \leq k, M(t)=0$ if $t<c$, and $M(t)=1.0$ if $t>k$, where $c \approx 0.400 \mathrm{~s}$ and $k \approx$ $0.850 \mathrm{~s}$ specified the start and end of motion integration. This equation is dimensionally balanced, not a regression equation. Data consistent with this equation are shown below in Fig. 6e.

Both the present and previous studies sought to determine how increases in size of the attentional field influence temporal processes for detecting visible target changes in dynamic displays. A main independent variable was the set size of attentionally relevant moving objects. ${ }^{4}$ Both studies measured detection rates by hazard functions. ${ }^{5}$

Two significant modifications of the present study involved the target signals and the response task. Target signals in the present study were sudden changes in object color or motion direction - temporally very different from the linearly increasing target motions of Lappin et al. (2016). Both subjectively and as described by the data, these momentary signals were very visible when they occurred but hardly noticeable afterward. A basic question was how these momentary signals might affect the temporal processes of detection. Another methodological modification was a comparison of two different response tasks. One task required a Simple response to any target change, and the other required Selective responses to only half of the display changes, which, not surprisingly, slowed the detection responses. Thus, the present study investigated the influence of both stimulus and response factors on temporal processes of target detection. The general question was how these processes may be affected by distributed attention and limitations of perceptual capacity.

\section{Approaches to perceptual capacity}

Perceptual capacity has been measured by several methods, involving different paradigms, assumptions, and terms. Two such methods are the following.

Applications of Shannon's Fundamental Theorem: Shannon (1948) established an upper bound on the rate at which any given channel can transmit information. The maximum rate of information transmission is a physical limit given by the channel's maximum rate of entropy. Because the brain is a physical system, its transmission rates might also be capacity-limited. Applications of Shannon's theory to human performance led to a new way of representing sensory input, perceptual processes, and behavioral output. Experiments typically varied the numbers of stimulus and response alternatives, and measured transmitted

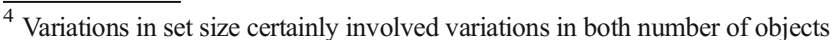
and spatial complexity. This study does not distinguish these two factors.

${ }^{5}$ RT distributions and hazard functions were all defined by the physical times after the target signal change until a response-including trials with no response, when targets were undetected. Analyses were based on the survival functions, $S(t)=1-F(t)=$ relative frequency of non-detection by time $t$.
}

information by the correlation between stimulus input and response output selections. Estimates of channel capacity were based on identification accuracy, not speed. Miller (1956) and Garner (1962) summarized the results of many such experiments, and found consistent averages around 2.5 bits for variations in a single dimension. Identifications of multi-dimensional everyday objects, however, have not seemed limited by a simple channel capacity. When Hyman (1953) applied Shannon's information measure to stimulus identification speeds, he found that three types of uncertainty - number of alternatives, relative probability, sequential dependency — had essentially equal effects. Mean RTs for 24 conditions correlated with uncertainty: $r^{2}=$ 0.92 , slope $=174 \mathrm{~ms} / \mathrm{bit}(5.75 \mathrm{bits} / \mathrm{s})$. Nevertheless, Shannon's fundamental theorem has been tested with only a few methods.

Multiple object tracking (MOT): Pylyshyn and Storm (1988) had observers track designated targets within ten identical randomly moving objects, responding rapidly to the test flash of a target while not responding to flashes of distractors. The authors concluded that observers were able to track up to five moving objects. Mean RTs ( $635-715 \mathrm{~ms})$ and detection misses $(\sim 2-14 \%)$ both increased, however, with the number (1-5) of targets. Subsequent studies of MOT (e.g., Alvarez \& Franconeri, 2007; Cavanagh \& Alvarez, 2005; Fehd \& Seiffert, 2010; Franconeri, Jonathan, \& Scimeca, 2010; Pylyshyn, 2004; Tombu \& Seiffert, 2008; Tran \& Hoffman, 2016) have tested target detections after the object motions stop. Results are usually similar to those for VWM: few errors for one to three targets, frequent errors for four targets, and few correct identifications of five targets. This nonlinear effect of the MOT set size on identification accuracy may differ qualitatively from roughly linear effects on speed and accuracy found by Pylyshyn \& Storm's (1988) test-flash method. MOT demonstrates clear perceptual capacity limits, but differences in capacity limits measured by MOT, VWM, RTs, and applications of Shannon's theorem have not been clarified.

\section{Measuring perceptual capacity with hazard functions}

Visual detection rates and capacity limits may be quantified by hazard functions, as in our previous study (Lappin et al., 2016). Extensive research by Townsend and colleagues (e.g., Altieri, Stevenson, Wallace, \& Wenger, 2015; Houpt \& Townsend, 2012; Townsend, 1990a; Townsend \& Alteri, 2012; Townsend \& Ashby, 1978; Townsend \& Eidels, 2011; Townsend \& Nozawa, 1995; Townsend \& Wenger, 2004a, b) has demonstrated the value of hazard function measures. In effect, RTs may be treated as an external factor, as system observation times. Coupled with factorial combinations of independent variables, hazard functions describe process characteristics not revealed by conventional RT measures (e.g., 
Little, Altieri, Fific, \& Yang, 2017; Luce, 1986; Townsend, 1990b).

Three functions pertinent to the present application are (a) the survivor function, $S(t)$; (b) the cumulative hazard function, $B(t)$; and (c) the hazard rates, $b(t)$ and $b^{\prime}(t)$. The response probabilities in these functions are defined on all target occurrences, including both missed detections and correct detection RTs. These functions are defined as follows:

(a) $S(t)=1-F(t)$, where $F(t)=p r(R T \leq t)$.

The survivor function is the relative proportion of targets not yet detected by response time $t$-i.e., the complement of $F(t)$, the cumulative distribution function of RTs.

(b) $B(t)=-\log _{2} S(t)$.

The cumulative hazard function, $B(t)$, measures the cumulative work - detection progress - up to time $t$. The conventional notation is $H(t)=-\ln S(t)$. In place of this notation and scale in natural $\log _{e}$, we have chosen to scale these functions by $\log _{2}$ - simply because this offers convenient terminology. Thus, $B(t)=H(t) / \log _{e}(2)$, which affects only the scale but not the meanings of the hazard functions and equations. Units of the cumulative function, $B(t)$, are in bits-where $B(t)=1.0$ designates the median RT, and each 1-bit increase reduces the survivors by $1 / 2$.

The derivative function, $b(t)$, measures the rate of change in the cumulative function $B(t)$, with units in bits/s. For continuous distributions, this "detection rate" (hazard rate, or "intensity function") is defined as the probability density at time $t$ conditioned on the survivors at that time,

(c) $b(t)=\lim _{\Delta t \rightarrow 0}[B(t+\Delta t)-B(t)] / \Delta t=[f(t) / S(t)] / \log _{e}(2)$

In practice, with discrete response frequencies, the detection rate functions may be estimated by discrete approximations of the local slopes of the cumulative functions:

$$
b^{\prime}(t)=[B(t+\Delta t)-B(t)] / \Delta t
$$

where $\Delta t$ may be an ephemeral variable determined by the times at which the survivor functions reach specified values. Here, detection rate functions were described at $\Delta t$ intervals corresponding to overlapping 20-percentile windows of the RT distribution-where $S(t)=95-75 \%, 90-70 \%, \ldots, 25-$ $5 \%$. Note that this formula quantifies the detection rate in the time interval $\Delta t$ immediately following time $t$, whereas the cumulative function, $B(t)$, is based on RTs $\leq t$. These running windows were found to yield effective estimates of the detection rates that balanced a tradeoff between temporal resolution and smoothness of the functions. Descriptive analyses of these detection rate functions in the present study were applied to the pooled RT distribution of all five participants within each of 16 (four set sizes $x$ two visual signals $x$ two response tasks.), treating the RTs as if produced by a single individual. Illustrations and further comments about these measures are given in the Results section.

The present experiment used these hazard functions to evaluate temporal processes in a task similar to Pylyshyn \& Storm's (1988) test-flash method for studying MOT. The visual displays were analogous to those used by air traffic control or military surveillance systems. The present hazard functions described temporal processes for visual signals and response choices that were qualitatively different from those in our previous study (Lappin et al., 2016). The results extended and validated this method for quantifying perceptual capacity limits.

\section{Method}

Participants Adult volunteers were recruited from the undergraduate students at Vanderbilt University. Eight paid volunteers (five females) were fully informed and practiced in the procedures, tasks, and performance objectives (fast and accurate), but were otherwise naïve to the purposes of the study and expected results. All participants were tested under Human Research Protections of Vanderbilt University and in compliance with the Belmont Report. Data were not analyzed for two potential participants - one of whom could not return after the first two sessions, and the other failed to perform with sufficient accuracy in the easiest conditions. Data from the other six were analyzed individually. The reported results do not include data for one of these six because (a) his performance was consistently slower, less accurate, and obscured by floor effects; (b) his testing was compromised by a 2-week absence during the experiment; and (c) he provided less data than the other participants.

Visual information displays Red, green, and white square objects that symbolized planes moved over a map including a stationary ship-like icon centered in a 27-degree display. Figure 1 shows a stationary image of the display. A principal independent variable was the Set Size of simultaneously visible moving objects - either 1, 2, 4, or 6 objects. Objects were either 0.66 or 1.07 degrees in width. All objects moved at a constant velocity in straight lines, moving into the display and later disappearing at the edge of the circle. The origins and destinations of these "flight paths" were randomly distributed around the 12 cardinal "clock-face" positions at the periphery of the circular region, as described in the caption of Fig. 1. The direction of every object was also indicated with a small bar (1.25 degrees) stemming from the center of the object. The sizes and directions of the moving objects were all randomly assigned within each display. Three different object colors- 

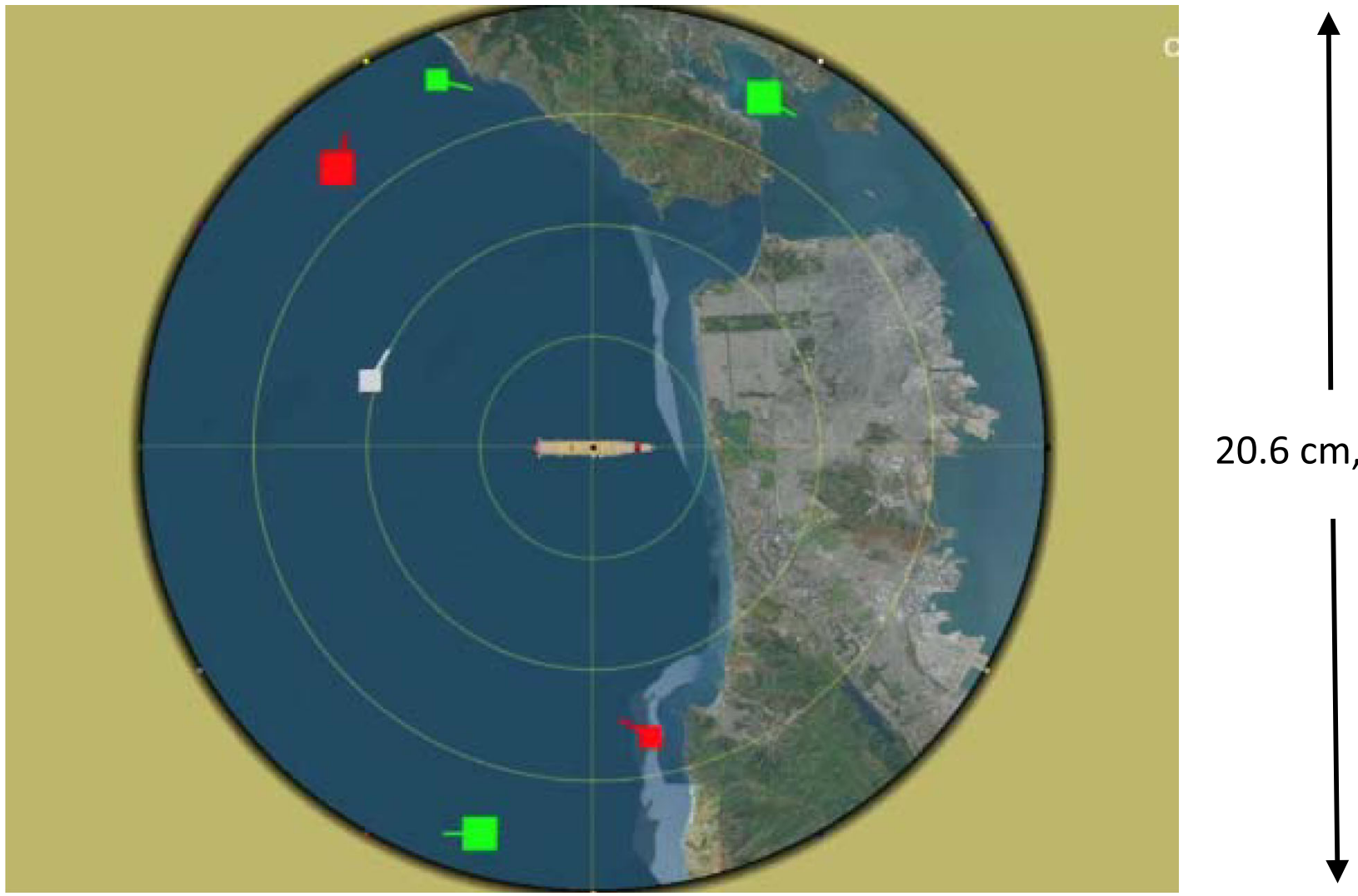

$20.6 \mathrm{~cm}, 27^{\circ}$

Fig. 1 A one-frame example of the moving visual displays. The depicted scene represented a "naval air warfare" scenario, in which the moving icons symbolized planes posing potential "threats" to a stationary shiplike icon in the center. During continuous 6-min observation periods, the display contained either $1,2,4$, or 6 moving objects, with that number constant throughout the observation period. These moving objects entered the circular display area from a randomly chosen one of 12 clockface positions around the periphery of the circular display area. They proceeded at a constant velocity $(1.3 \mathrm{deg} / \mathrm{s})$ toward one of six alternative destinations at clock-face positions, three to each side of the starting position of the flight path, $\pm 30^{\circ}, 45^{\circ}$, or $60^{\circ}$ from the tangent at the start position. (E.g., if the start position was at 12 o'clock, then the potential destinations were at 10, 9, 8, 2, 3, and 4 o'clock, with equal probability.) The object disappeared when it reached its destination, and was immediately replaced by another object appearing with equal likelihood at one of the other 11 start positions. Two simultaneously visible objects never had the same flight path. Perception of motion direction was aided by the orientation of a short line attached to each object to specify its direction of motion. Three equally likely object colors were green, red, and white, representing respectively friend, foe, and unknown. The objects also had one of two equally likely sizes $\left(0.66\right.$ or $\left.1.07 \mathrm{deg}^{2}\right)$. In pilot studies, with different participants, the larger size symbolized lower altitude, but the size was meaningless and irrelevant to the task in the present study

about 15 changes per min. Changes did not occur in either the outer $0.95 \mathrm{deg}(1.3 \mathrm{~cm})$ or inner $0.95 \mathrm{deg}$ of the display. This restriction reduced the number of changes that were presented especially for the 1-Object displays, but this variation in probability did not discernably affect performance.

Color changes were equally likely from any color to one of the two alternatives. Direction changes were an abrupt change of approximately $90^{\circ}$ from the linear tangential path in the outer annular region to a radial direction toward either the ship-like icon at the center or toward the perimeter. Motion toward the ship was said to constitute a "threat" and motion away from the ship was a "nonthreat".

\footnotetext{
${ }^{6}$ Two pilot studies evaluated potential effects of uncertainty about the dimension (color, size, motion direction) of change by comparing conditions with 1 , 2 , or 3 changeable dimensions. When we compared the effect of this dimensional uncertainty on detecting changes in a given dimension (e.g., motion direction), we found only small and inconsistent effects of dimensional uncertainty. Those effects were judged insufficient for further investigation.
}

Simple and Selective response tasks Two detection tasks assessed effects of the response decision. For both tasks, participants pressed the keyboard spacebar to report the relevant target changes. The Simple detection task required a response 
to any change. The Selective task, however, required a response to only half of the changes, only to those corresponding to an increase in "threat" to the central ship. Threat changes were motion direction toward the ship but not away from the ship, or a color change from green to red, white to red, or green to white, but not red to green, red to white, or white to green. Response time was the time between the display change and the participant's press of the spacebar. Because the displays and detection task were continuous, there were no separate non-target trials, and no explicit response for the absence of a target change. Responses immediately following a target change by more than $200 \mathrm{~ms}$ and less than $3 \mathrm{~s}$ were coded as correct detections and responses that did not follow a target change were coded as false alarms. Responses to nontarget changes in the Selective task were coded as incorrect responses. Target changes that did not elicit a response within $3 \mathrm{~s}$ were coded as misses (RTs beyond $3 \mathrm{~s}$ were less than $0.2 \%)$.

Procedure Participants were tested individually in a small testing room in the Psychology Department laboratory at Vanderbilt University. Experiments were controlled by Apple Mac mini computers with 2.5-GHz Intel Core equipped with DELL E1909W monitors at 1440X900 resolution and 60 Hz. Viewing distance was $43 \mathrm{~cm}$, unconstrained. Testing software was custom made by engineers at Vanderbilt University's Institute for Software Integrated Systems (details of the software are available by request from the first author). Prior to data collection, all participants practiced the experimental tasks to an apparent asymptotic level, during one or two practice sessions. Each testing session was $1 \mathrm{~h}$, and included 6 or 7 blocks of 6-min duration. Different participants completed different numbers of blocks and sessions dependent on scheduling limitations. All trials within a block tested a single set size, either 1, 2, 4, or 6 objects, and a single task, Simple or Selective. Within a block, Color and Direction changes occurred randomly. Blocks of all Set Sizes were counter-balanced and then repeated. Half the participants completed the Simple task first, while the other half completed the Selective task first. More sessions were devoted to the Selective task to compensate for the smaller number of responses per block. Summed over participants, the total trials for each condition ranged from 506 to 925 for Simple detections and from 287 to 549 for Selective detections, with smaller numbers for smaller set sizes.

\section{Results and Discussion}

Cumulative hazard functions Responses were accumulated into RT distributions separately for each participant, task, set size, and type of visual change. Cumulative hazard functions, $B(t)$, showing temporal processes and variations between individual participants, are shown in Fig. 2. The slopes of these cumulative hazard functions correspond to the time-varying rates of target detections, in bits/s. Differences in target detections in different conditions are readily apparent. These cumulative hazard functions were generally consistent among the individual observers, although larger differences occurred for Color than for Direction changes and larger for Selective than for Simple detection responses.

Temporal characteristics of visual target detection The temporal processes are described by the detection rates, $b^{\prime}(t)$, shown in Fig. 3. These detection rates are discrete estimates of the changing local slopes of the cumulative functions in Fig. 2-governed by the time intervals between increases in $B(t)$ associated with $5 \%$ decreases in survivor rate. That is, these detection rates, $b$ ' $(t)$, measure the 'intensity' or 'power' of target detections at successive points in time.

A salient feature of these detection rate functions is their shape - resembling a peak or hill, with a clear rise and fall in detection rates over a brief time window of about $1 / 4 \mathrm{~S}$ for detecting the visual change signals. The hill-like shape of these detection rate functions was very different from the shape of the corresponding functions found by Lappin et al. (2016), as shown in Fig. 6e. Differences in the amplitudes, latency, and spread of these detection rate functions may also be seen in comparisons among the four panels of Fig. 3 . Differences between the functions in panels Fig. $3 \mathrm{a}$ and $\mathrm{b}$ indicate that color changes were detected sooner and were more sharply peaked in time than detections of direction changes. Differences between the functions in the upper and lower panels, involving differences between the Simple and Selective tasks, also indicate that the selective responses were slower and less intense (lower amplitude) than the simple detection responses. Analyses below will examine these effects of the visual signal and response task.

Cumulative target detections at given times An important question concerns the combined influence of set size, visual signal, and response task on the temporal detection process. The surprising result, shown in Fig. 4, was that at given times, especially early in the process these three independent variables had approximately independent influence on target detections. Figure 4 evaluates cumulative target detections, $B(t)$, at three specific times encompassing the largest variations in detection rates.

Figure 4 also shows the predicted performance values, $B(t)$, for set sizes 2, 4, and 6, where the predicted values at each of the three times are the vector products of three independent factors plus the total mean - for the effects of set size, Color vs. Direction target signals, and Simple vs. Selective response tasks. Because the obtained performance for set size 1 clearly did not fit the same general relationship as the three larger set 
Set Size



Response Time (ms)

Fig. 2 Cumulative hazard functions for each participant in each condition. The slopes of these functions estimate the hazard rates (bits/s), which measure the rates of information processing at given times. The + symbols in each graph are values for each of the five participants, and the lines connect the means. Low values of $B(t)=0.25$ and 0.50 bits designate hit rates of $15.9 \%$ and $29.3 \% ; B(t)=1.0$ designates the median RT (based on all target presentations, including both hits and misses); and each unit increase in $B(t)$ reduces the proportion of undetected survivors by one half-yielding hit rates of $75,86.7,93.75,96.9$, and $98.4 \%$ for $B(t)=2,3,4,5$, and 6 bits, respectively 


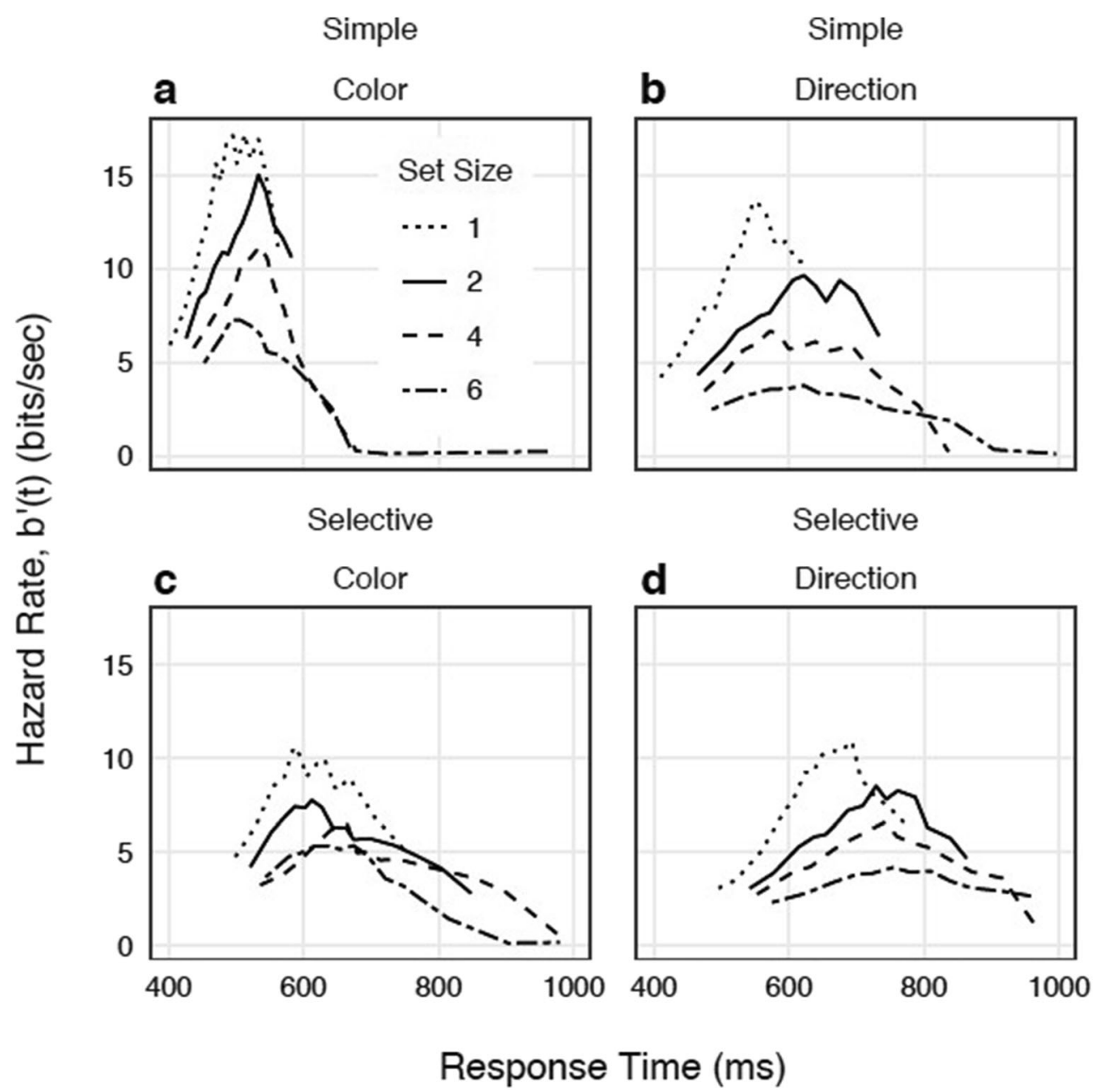

Fig. 3 Detection rate functions for each of the 16 conditions. These functions were computed from a single distribution of the pooled responses of all five participants, treating the combined RT distributions

sizes, predictions for set size 1 are not included in Fig. $4 .^{7}$ The four vector components for the predictions at each time are Mean: $B(t)=\{0.64,1.26,1.86\}$ for RTs $\leq\{600,700,800\}$; Set Size for $n=\{2,4,6\}:\{1.38,0.94,0.68\}$ at all three times; Signal \{Color, Direction $\}:\{1.40,0.60\}$ at $600 \mathrm{~ms},\{1.28$, $0.72\}$ at $700 \mathrm{~ms}$, and $\{1.18,0.82\}$ at $800 \mathrm{~ms}$; Response $\{$ Simple, Selective $\}:\{1.62,0.38\}$ at $600 \mathrm{~ms},\{1.39,0.61\}$ at $700 \mathrm{~ms}$, and $\{1.27,0.73\}$ at $800 \mathrm{~ms}$.

As may be seen, the predicted and actual performance values corresponded closely at 600 and $700 \mathrm{~ms}$, but significant deviations from the predicted values occurred at $800 \mathrm{~ms}$. Deviations (standard deviations of the binomial distribution

\footnotetext{
${ }^{7}$ When the set size 1 predictions were included, deviations between predicted and obtained values were statistically significant at all three times. Differences in performance for set size 1 vs the larger set sizes may also be seen in Figs. 3 and 5. Even so, the average correlation between predicted and actual performance at all three times when the set size 1 data were included was $R^{2}(d f=38)$ $=0.972$.
}

as if for a single person. Fifteen data points in each function were computed for running $20 \%$ intervals at successive $5 \%$ points in the $S(t)$ survival functions - at $95-75 \%, 90-70 \%, \ldots .25-5 \%$

of response frequencies, 506-925 trials for Simple responses and 287-549 for Selective responses) between predicted and actual values at the times 600,700 , and 800 , respectively, were RMS $(d f=8.67)= \pm 1.05(p<0.298), \pm 1.43(p<$ $0.153)$, and $\pm 2.35(p<0.019)$, and the average for all 36 data points was RMS $(d f=26)= \pm 1.70(p<0.089)$. The greater deviation of the obtained performance values at $800 \mathrm{~ms}$ reflects a 4-way interaction (time $\mathrm{x}$ set size $\mathrm{x}$ target signal $\mathrm{x}$ response task). The largest deviations occurred for the set size effects in the Selective Color condition and the Simple Direction condition at set size 6. Related evidence of performance variability in the Selective Color condition may also be seen in Figs. 2, 3, and 6. Reasons for the greater variability and greater deviations from predicted independence in the Selective Color detections are not clear. In general, however, the obtained cumulative hazard functions reflect approximately independent influence of the three independent variables in this study. Interestingly, that independent influence is clearer at earlier rather than later response times. 


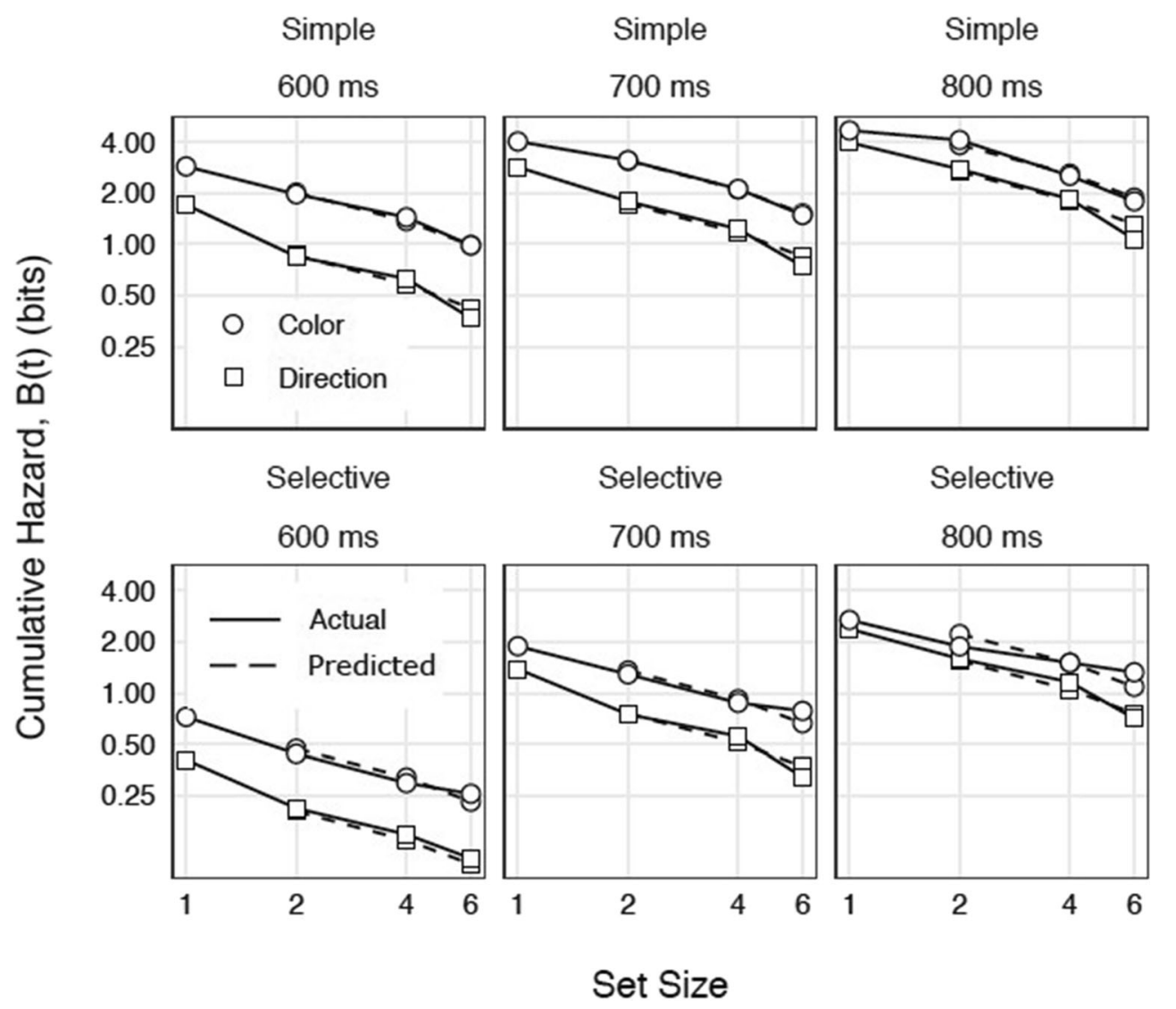

Fig. 4 Target detection progress at three points in time, as a function of attentional set size for two visual signals and two decision tasks. The 'Actual' values connected by solid lines are averages for five observers. The 'Predicted' values for set sizes $n=2,4, \& 6$, connected by dashed lines, are the products of three independent factors plus the total mean at each time - for the effects of set size, Color vs. Direction signals, and Simple vs. Selective responses. The estimated set size effects were based on the slope of a linear correlation with $\log (n)$ that was the same at each of

Proportional hazard functions and detection rates Figure 5 shows temporal variations in the relative values of cumulative hazard functions for the main effects of the three independent variables in Fig. 4. Figure 5c, d are especially noteworthy, indicating that the three hazard functions for set sizes 2, 4, and 6 maintained similar proportional values over time. Figure $5 \mathrm{~d}$ shows effects similar to those in panel (c) but computed on the independent detection rates, $b^{\prime}(t)$, for three successive $100-\mathrm{ms}$ intervals. The constant proportionality of these three hazard functions is remarkable given that the underlying response rates were quite different and changing, as seen in Fig. 3. Moreover, the Color and Direction signals yielded different detection rates with different temporal characteristics, as seen in Fig. 5a, b as well as Figs. 3, 4, and 6. Despite these differences in detection rates for different signals at different times, increases in set size produced consistent divisive effects on the detection rates. Figure $5 \mathrm{c}$, d also shows that the temporal detection process for set size 1 was different from that for the larger set sizes. The earlier rise in these times and predicted the relative set size effects for all 36 points. The effects of the Color vs. Direction signals and the Simple vs. Selective responses, however, changed over time and were estimated independently at each of the three times. With a logarithmic scale of the vertical axis, the functions for predicted set size effects are not linear, but the 12 functions for set size are all parallel and the four functions at each time are equally spaced

detection rate for set size 1 is not surprising; the important surprise is that similar temporal differences were not observed for increases in set size from 2-6. Analyses and interpretations of these proportional detection rates continue below, graphically and statistically.

\section{Detection rate functions reflect visual and response processes} Different visual signals yielded different detection rate functions, $b^{\prime}(t)$, differing not only in amplitude but also shape and temporal location. Such effects on the shape and location of these functions may be seen in Fig. 3, but those effects are more visible when the amplitudes are adjusted to compensate for differences due to set size. Figure $6 a$ and $b$ show the adjusted functions for Simple Color and Direction detections, and 6(c) and (d) show the adjusted functions for Selective Color and Direction detections. The underlying data are the same as in Fig. 3, but here the amplitudes for set sizes 2, 4, and 6 have been multiplied by factors based on the slope of the linear regression of $B(t)$ on $\log ($ Set Size $)$, which approximated 

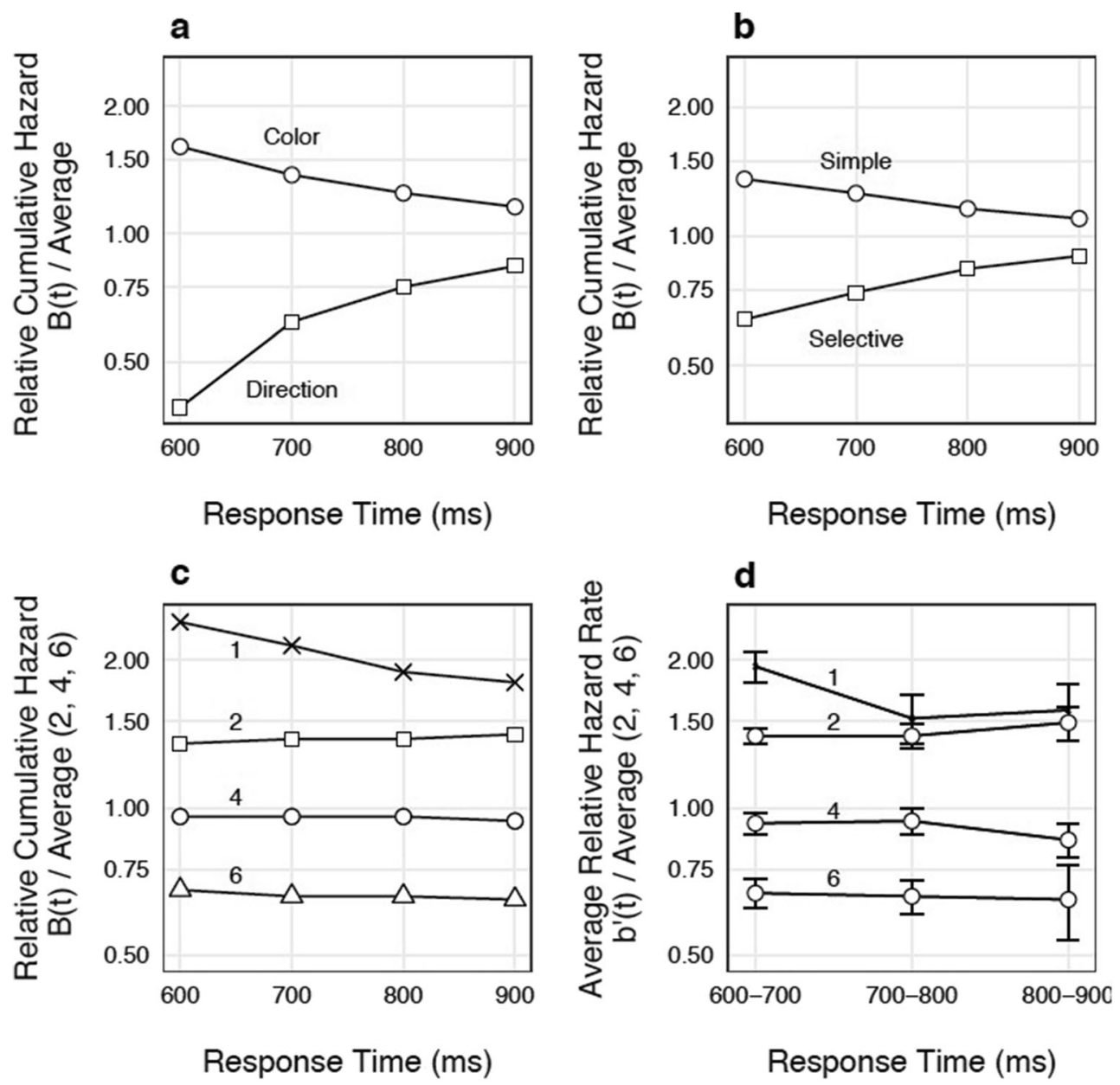

Fig. 5 Relative hazard functions for the main effects in Fig. 4. Panels a and $\mathbf{b}$ show the relative main effects for Color vs. Direction targets and Simple vs. Selective responses as functions of time. Each cumulative hazard value, $B(t)$, was averaged over the other variables and that average $B(t)$ value was then scaled relative to the average for the two conditions in each panel. The relative values at each time sum to 2.0. Panel $\mathbf{c}$ shows the relative main effects for Set Size, scaled relative to the average for set sizes 2, 4, and 6. The relative values for Set Sizes 2, 4,

$\left(R^{2}=0.97\right)$ the set-size effects for all 48 points in Fig. 4 , including those for set size 1 . Thus, the amplitudes for set sizes 2, 4, and 6, have been multiplied, respectively, by 1.35 , 2.09, and 3.08. Effects of these amplitude adjustments are more uniform for the Simple response tasks, in panels (a) and (b), than for the Selective responses, in panels (c) and (d). Effects of set size were least consistent for the Selective Color, in panel (c), as also seen in Figs. 2, 3, and 4. Variability of the set size effects in the Selective response tasks suggests that delayed responses in that Selective response task might have involved working memory for the Color changes. Memory probably had less of a role in detecting Direction changes, as the radial target directions were visible after the change.

The significant influence of visual signals on detection rates is also seen by comparison with data from our previous study (Lappin et al., 2016), in Fig. 6e, where detection rates

and 6 at each response time sum to 3.0. Panel $\mathbf{d}$ shows the average relative detection rates for successive 100-ms intervals: $b_{n}^{\prime}(t) / A v e\left[b_{n}^{\prime}(t)\right]$, where $n$ $=\{2,4,6\}$, and $b^{\prime}(t)=[B(t+0.1 s)-B(t)] / 0.1$. These relative detection rates were computed individually for each participant in each condition, prior to computing the averages in panel d. Error bars are \pm 1.0 S.E.M, based on 20 independent values for each data point. The detection rates at these three successive time intervals were independent, unlike the cumulative values in panels $\mathbf{a}, \mathbf{b}$, and $\mathbf{c}$

were found to be inversely proportional to set size. When the detection rates in Experiment 1 of that study were increased in proportion to set size, the resulting adjusted detection rate functions were all very similar. Importantly, the detection rate functions in the previous and present studies had very different temporal structures, and these temporal differences are attributable to the temporal structures of the optical signals. Target signals in the present study were optically defined at the moment of change, and, for Color changes at least, were indistinct after the change. In contrast, target signals in the previous study were approximately linear trajectories differing from a random-motion background, with the target-background differences becoming increasingly visible over time. Accordingly, the detection rate functions in panel (e) reflected a visual process of motion integration, unlike the rise and fall of change detections in the present study. 


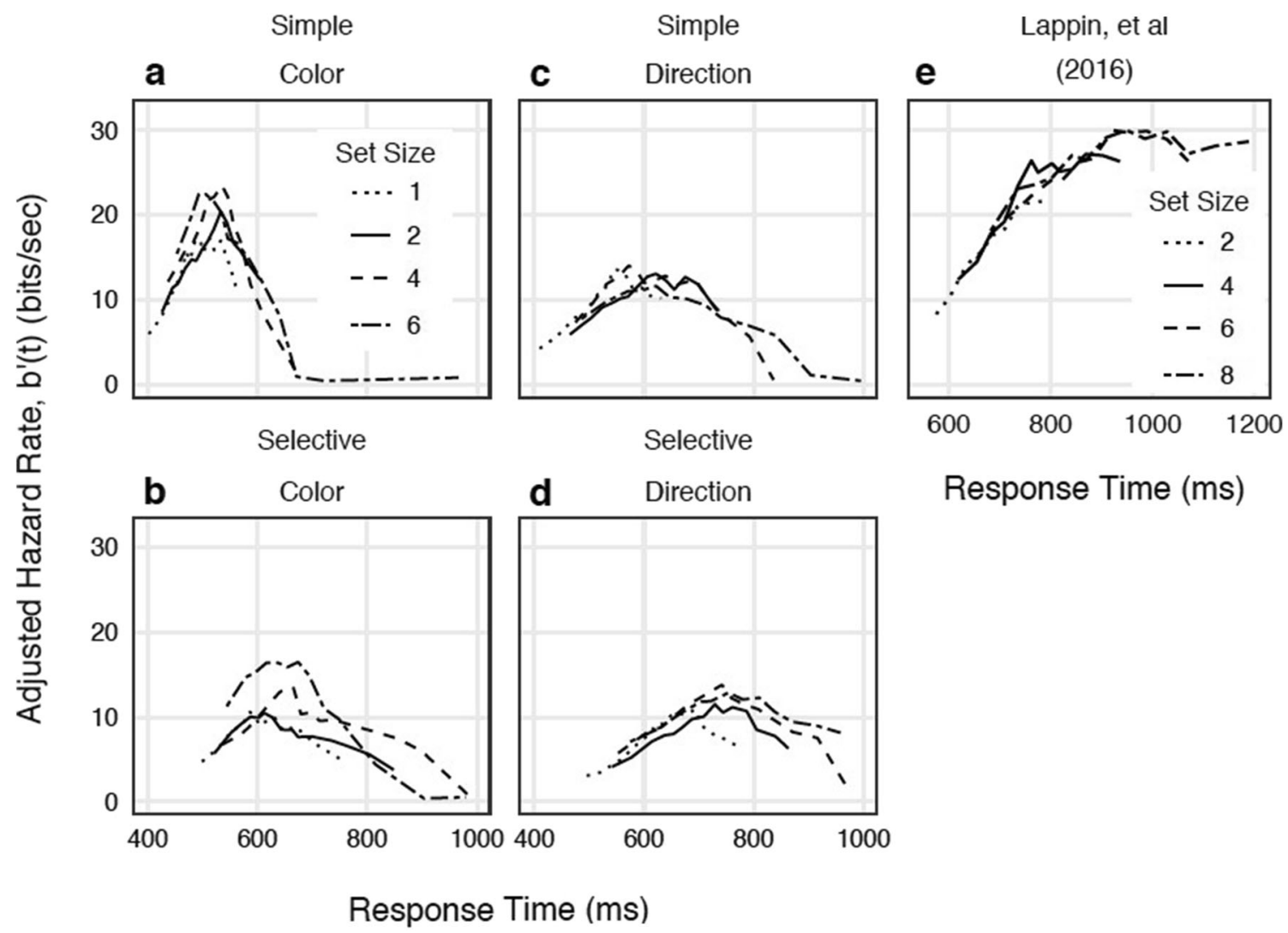

Fig. 6 Detection hazard rates adjusted to compensate for the divisive effect of the attentional field. This adjustment increases visibility of the similar shapes of the hazard rate functions for different set sizes and clarifies the effect of the target signals on the shapes of the detection rate functions. The graphs in panels $\mathbf{a}, \mathbf{b}, \mathbf{c}$, and $\mathbf{d}$ correspond to the data in Fig. 3, but here the detection rates for set sizes 2, 4, and 6 in each graph have been multiplied by $1.35,2.09$, and 3.08 , respectively (these factors were computed from the linear correlation between $B(t)$

and $\log _{2}$ (Set Size) that approximated the relative set-size effects among the 48 data points in Fig. 4.). The data in panel e were replotted from the results of Lappin, Morse, \& Seiffert (2016), resembling Fig. 3b of that study, in which detection rates were found to be inversely proportional to set size (similar to the functions in the other four panels, this replotted graph specifies adjusted detection rates for overlapping $20 \%$ windows at successive 5\% intervals (from 90 to $10 \%$ ) of the survivor function)

Statistical tests of proportional hazard functions Tests of the hypothesized temporal constancy of relative hazard functions for set sizes 2, 4, and 6 were based on the predicted response frequencies corresponding to predicted relative detection rate functions. Predicted detection rates, $b^{\prime}(t)$, were estimated as if the relative rates for the three set sizes $(2,4,6)$ were equal for three time intervals (600-700, 700-800, 800-900)-i.e., 3 x 3 data points with five parameters ( 3 time intervals +2 relative set size factors), $d f=4$. These computations were done for each of the five participants, though the number of free parameters was reduced by using the same two estimated relative set size parameters for all five participants, yielding 17 parameters ( 5 observers x 3 time intervals +2 relative set size factors) for 45 data points, $d f=28$. Predicted hazard rates were converted to response frequencies, and deviations between observed and predicted frequencies were scaled in standard deviation units for the binomial distribution: $\sigma_{b}=\sqrt{ }[N p(1-$ p)], where $N$ is the number of non-detected survivors at the start of a given time interval, and $p$ is the predicted probability of survival in that interval. These deviations between the

Table 1 RMS deviations from predicted temporal constancy-over three successive 100-ms intervals from $600-900 \mathrm{~ms}$ - of relative hazard rates for varied set sizes. Deviations are scaled relative to the standard deviation of the binomial distribution. For normally distributed deviations, critical values for confidence interval widths of $60 \%, 75 \%, 90 \%$, and $95 \%$ are, respectively, $R M S=0.84,1.15,1.64$, and 1.96. An asterisk (*) marks values beyond the $90 \%$ interval, and two asterisks (**) mark a value beyond the $95 \%$ interval. (Deviations between obtained and predicted values may be positive or negative, while $R M S$ values are equivocally \pm .)

\begin{tabular}{lcclllll}
\hline Condition & \multicolumn{3}{l}{ Participants } & \multicolumn{3}{c}{ RMS average } \\
\cline { 2 - 6 } & A & B & C & D & E & \\
\hline Simple Color & 0.98 & 1.31 & $2.16^{* *}$ & 0.86 & 0.94 & 1.34 \\
Simple Direction & 0.91 & 1.19 & 1.29 & 1.32 & 1.31 & 1.21 \\
Selective Color & $1.70^{*}$ & 0.80 & 1.56 & 1.61 & 1.51 & 1.49 \\
Selective Direction & $1.70^{*}$ & 1.41 & $1.64^{*}$ & 0.99 & $1.73^{*}$ & 1.52 \\
RMS average & 1.37 & 1.20 & $1.70^{*}$ & 1.23 & 1.40 & 1.39 \\
\hline
\end{tabular}


observed and predicted values were evaluated for each of the five participants in each of the four target detection conditions. These standard deviations are summarized in Table 1 by root mean square $(R M S)$ deviations for each participant and each condition. Treating the deviations as normally distributed, confidence intervals were approximated by the normal distribution (computational details are described in the Supplementary Material section).

As may be seen, $R M S$ deviations between predicted and observed detections were comparable to those associated with the binomial distribution. The overall total $R M S$ deviation between obtained and predicted detection response frequencies was $R M S= \pm 1.39 S D, p=0.16$ in the two-tailed normal distribution. Most of the 20 deviations were between 1.0 and 2.0 SD. Six were less than $1 S D$, and one was greater than 2 $S D$; five were outside the $90 \%$ confidence interval, and one was beyond the $95 \%$ interval. The largest deviation involved ceiling effects for the best performing participant in the easiest condition - where the cumulative hit rate at $800 \mathrm{~ms}$ was $97 \%$ with only four survivors and no responses in the 800-900-ms interval, yielding a spurious hazard rate of $b^{\prime}(t)=0$ for the 800-900-ms interval.

The obtained RMS deviations were slightly larger than expected by the binomial distribution - attributable in part to the inherent variability of hazard rates and to a magnifying transformation from errors in estimated hazard rates to standard deviations of response frequencies, especially as survivor frequencies diminish at high performance levels. The RMS deviations in Table 1 were larger than expected under the null hypothesis, but not so large as to confidently reject that hypothesis.

These results along with those in Fig. 5c, $\mathrm{d}$ are inconsistent, however, with the alternative hypothesis that larger set sizes delayed or lengthened the detection processes seen in Figs. 3 and 6. If larger set sizes added to the process times for detecting target signals, then the set size effects would vary with RT. Instead, increased set sizes had essentially the same divisive effects for faster and slower responses, reducing the rates but not the durations of the detection processes.

Similar set size effects for different target signals and response tasks RMS deviations analogous to those in Table 1 were also used to evaluate whether variations in set size produce the same proportional hazard rates in different conditions. Four different conditions involving color vs. direction target changes and simple vs. selective responses produced substantially different hazard rates at any given time, as seen in Figs. 3, 4, and 5. The latency and temporal spread of the detection rates also differed among these conditions. Thus, the effects of set size might well depend on the visual signals and response decisions.

We evaluated such interactive effects by computing $R M S$ deviations between obtained and predicted numbers of target detections for each set size (2, 4, and 6) and time interval (600-700, 700-800, and 800-900 ms) in each condition based on the average proportional hazard rates for two given conditions. The tested hypothesis is that the hazard rates for set sizes 2,4 , and 6 had the same proportions for both conditions and were also constant over time. Table 2 gives the pair-wise deviations for the six pairs of target detection conditions along with the deviations within a single condition.

Between-participant variations in each condition add to the $R M S$ deviations in Table 2 -in the single-condition values in the main diagonal and in the pair-wise values above the diagonal. Between-participant variability was especially pronounced for the Selective Color detections, where all deviations within and between this condition were greater than 2.0 standard deviations. Thus, the pair-wise deviations must be evaluated relative to the variability within conditions. The relevant values are below the main diagonal.

Underlined bold italicized values below the diagonal of Table 2 are ratios of the two-condition $R M S$ deviations relative to the $R M S$ average of the two single-condition deviations. As may be seen, these ratios averaged only 1.07 . Thus, variations
Table 2 Pair-wise tests of similarity of proportional hazard rates in three 100 -ms intervals for set sizes 2, 4, and 6 in two conditions with different target signals and response tasks. Entries in bold above the main diagonal are $R M S$ deviations of observed hit rates from predictions based on constant proportional hazard rates in the two given conditions and over the three time intervals. As in Table 1, the deviations are scaled by the standard deviation of the binomial distribution. Predicted relative hazard rates were the average relative values for the two conditions, averaged over the five participants in each condition. Numbers of non-detected 'survivors' were totaled across the five participants. Entries in the main diagonal quantify deviations from proportional hazard rates within a single condition. Between-participant variability in each condition adds to these $R M S$ deviations, so the pair-wise values should be evaluated relative to the single-condition values in the main diagonal. Underlined bold italicized entries below the main diagonal are ratios of the between-condition deviations (above the diagonal) relative to the $R M S$ average deviations of the two single-condition deviations.

\begin{tabular}{|c|c|c|c|c|}
\hline & Simple Color & Simple Direction & Selective Color & Selective Direction \\
\hline Simple Color & 1.23 & 1.37 & 2.50 & 1.48 \\
\hline Simple Direction & $\underline{1.04}$ & 1.39 & 2.56 & 1.39 \\
\hline Selective Color & $\underline{1.13}$ & $\underline{1.13}$ & 2.89 & 2.20 \\
\hline Selective Direction & 1.15 & 1.01 & 0.98 & 1.35 \\
\hline
\end{tabular}




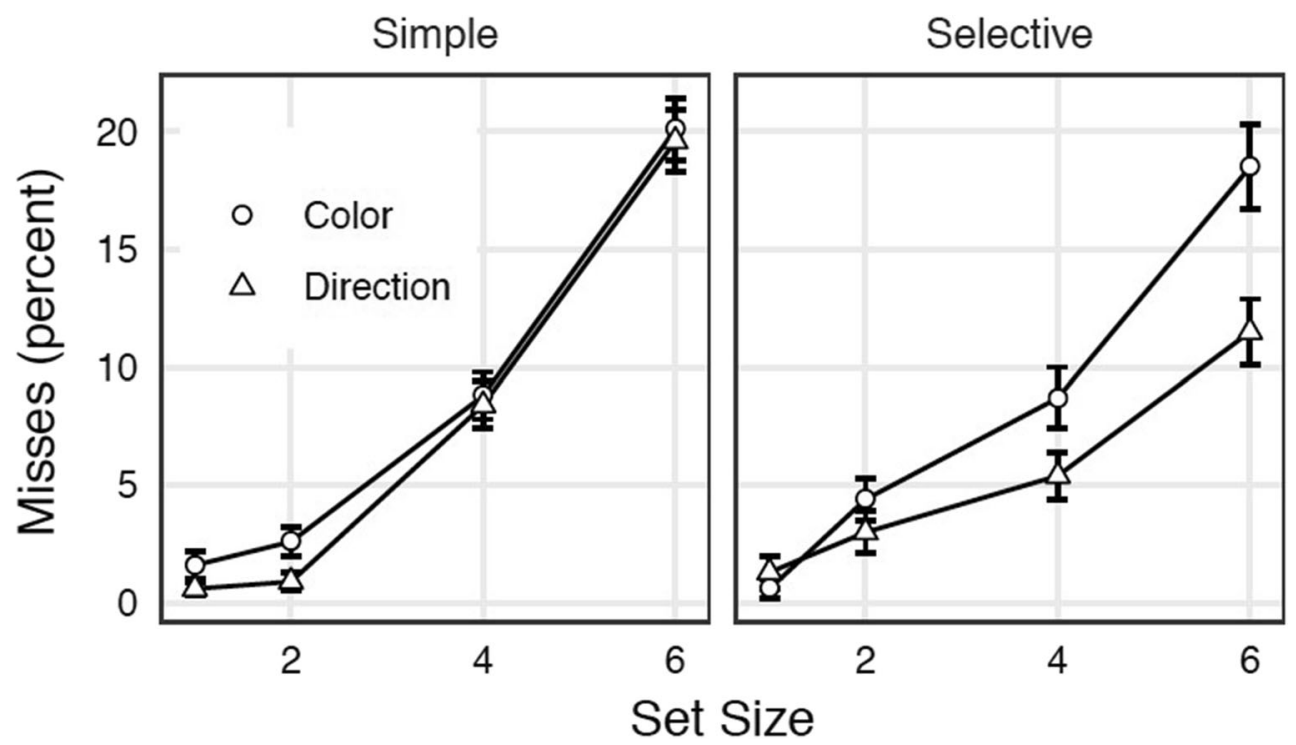

Fig. 7 Average percentages of missed target detections. Response times greater than $3 \mathrm{~s}$ were counted as misses, but such slow responses were rare. The error bars specify \pm 1 standard deviation of the binomial

distribution, based on the total trials for all five participants, ranging from 506 to 925 trials in the Simple response condition and 287-549 trials in the Selective condition.

in set size produced similar proportional hazard rates in different conditions as well as over time. This statistical evidence reinforces that in Figs. 4 and 5 for the same conclusion.

Detection failures Target detections were time-dependent. The obtained temporal processes of target detection were well described by the hazard functions, but missed detections add evidence that spreading attention reduces detection of easily visible targets. Figure 7 shows the percentages of missed target detections averaged over the five participants for each of the 16 conditions. Responses that occurred more than $3 \mathrm{~s}$ after the target occurrence were counted as misses. Very slow responses were ambiguous, because they were rare and because another target change was increasingly likely after $3 \mathrm{~s}$.

Detection misses increased substantially with set size, roughly compatible with the effects described by hazard functions. The primary differences between the detection data in Fig. 7 and the RT-based hazard functions in Fig. 4 involve the effects of the Color vs. Direction signals and the Simple vs. Selective response tasks. Both of these variables had much smaller and even opposite effects on detection accuracy and the hazard functions.

The reversal in relative detectability of the Color and Direction signals, as described in Fig. 7 vs. Fig. 4, probably results from the target motion directions being visibly displayed after the abrupt direction change, whereas color changes were not directly visible after the change, distinguished only by memory of the immediately preceding color. Why this visual advantage for the Direction signals was more evident in the Selective task is not clear, but is probably related to slower responses in the Selective task.

The very different effects of the Selective response task on hazard functions and missed detections suggests that while the
Selective responses were slower they did not lose information about the target signal. In any case, these two dependent measures probably reflect different processes that are differently affected by the size of the attentional field.

Detection failures probably occurred at the final stages of processes described by hazard functions, where VWM may have a larger role. Important theoretical questions remain to be answered about functional relations between the perceptual capacity limitations described by the hazard functions and the more memory-dependent detection failures described in Fig. 7. Are real-time perception and working memory separate serial processes, two temporally overlapping processes, or different aspects of the same process? How does the limited channel capacity in the present study interact with the limited span of VWM? New experiments are needed to clarify these basic issues.

False alarms in Selective response conditions Procedures, training, and instructions were designed to minimize incorrect responses to changes defined as nonthreats in the Selective condition. Occasional response errors were inevitable, of course, but were sufficiently infrequent relative to the hit rates that they did not alter interpretations of the hazard functions. For Set Sizes 1, 2, 4, and 6 respectively, response rates to nonthreat changes were $0.072,0.114,0.115$, and 0.094 , with corresponding discrimination measures $d^{\prime}=3.85,2.97,2.70$, and $2.39\left(d^{\prime}=z(H R)-z(F A R)\right)$.

\section{General discussion}

This study sought to determine how increases in size of the attentional field influence temporal processes for detecting 
visible target changes in dynamic displays. In general, the attentional field (set size) was a major determinant of the speed in detecting any given target event. The principal finding was that Increasing the size of the attentional field produced consistent reductions in the rates for detecting and responding to visual target signals. Detection rates decreased proportionally with log set size - and these reductions in detection rates were, to a good approximation, constant over time and over different target signals and response conditions. For that reason, these findings suggest that the rate of visual perception has a limiting channel capacity. As a result, spreading attention divides the rates of perceptual processes.

Response time hazard functions A critical aspect of the methodology was the use of RT hazard functions for measuring the momentary rates and temporal process of visual target detection. Because this method has not been widely used, and because some aspects of the method depart from convention, several features should be noted:

First, the dependent measures of performance were not RTs as such, but instead were derived from the changing momentary response rates - i.e., hazard rate functions - which evaluated detection performance at given times. In effect, the RTs were independent observation parameters, as times at which detection performance was evaluated. A subtle but important point is that measures of hazard functions and measures of RTs usually yield different structures of relative performance for variations in task conditions. The lawful structure of the present results was revealed by hazard functions and not by RTs as such. Supplementary Material below compares RT and hazard function measures of the present results. It also explains why RTs are not always suitable measures of temporal processes, and why they were not appropriate for the present results.

Second, hazard functions are ratio scale measures of relative processing speed-for comparing different times in the process for a particular input signal and output response, and for comparing different processes at the same times. Those ratio scale measures were critical to the present results as well as those of our previous study (Lappin et al., 2016). Both studies found that increases in the attended set size had constant divisive effects on detection rates - effects that were constant over time and for different target signals and response tasks.

Third, differences in perceptual process rates do not imply differences in process latency or duration. Set sizes 2, 4, and 6 had substantial divisive effects on detection rates, but those effects were essentially constant over time, without shifting the temporal locations of the minima and maxima of the detection hazard rate functions. RTs would not reveal this time-invariant influence of the attentional field.
Continuous information flow The conception of RTs as sums of sequential process times is intuitively plausible and once was widely accepted (e.g., Donders, 1868/1969; Sternberg, 1966, 1969). Townsend and colleagues (e.g., Townsend \& Ashby, 1983; Townsend, 1971, 1972, 1990b; Townsend \& Wenger, 2004a) have shown, however, that limited-capacity parallel processes and serial-process architectures can generally produce the same RT distributions. They have also shown that serial and parallel processes can be distinguished by survivor and hazard functions (see Little et al., 2017; Townsend \& Wenger, 2004a).

Hazard functions in the present study point to a type of parallel processing known as "continuous flow" (Eriksen \& Schultz, 1979). Eriksen and colleagues found both behavioral and physiological evidence that responses to a target stimulus can be concurrently influenced by information in the surrounding field (Coles, Gratton, Bashore, Eriksen \& Donchin, 1985; Eriksen, Coles, Morris, O’Hara, 1985; Eriksen \& Hoffman, 1973; B.A. Eriksen \& Eriksen, 1974; Eriksen, 1995; Eriksen \& Schultz, 1979; Gratton, Coles, Sirevaag, Eriksen, \& Donchin, 1988; Eriksen \& St. James, 1986; St. James \& Eriksen, 1991; Miller, Riehle, \& Requin, 1992; Fournier \& Eriksen, 1990). The surrounding visual information either facilitated or competed with a target response, depending on whether flanking stimuli were compatible or incompatible with the target. McClelland's (1979) "cascade model" provided converging theoretical support, with continuing information flow from earlier to later processes in temporally overlapping stages.

Continuous information flow from multiple parallel processes was clearly exhibited by the results in Figs. 3, 4, 5, and 6. Variations in set size, visual signals, and response task all exerted concurrent rather than sequential influence on the detection process.

On the other hand, the results in Figs. 4 and 5 do not exhibit the contingent process interactions posited by McClelland's model. Instead, at any given time, three task variables exerted mutually independent influence on target detections. The process independence exhibited in Fig. 4 replicates and extends analogous results of Lappin et al. (2016).

Complexity of the attentional field The number of objects in the attentional field was a major determinant of speed and accuracy in detecting a particular target event. Quantifying the information load of the attentional field, however, is a challenging fundamental problem. Both the present and preceding (Lappin et al., 2016) studies evaluated the attentional field in terms of its effects on detection rates.

Detection rates in our previous (2016) study were inversely proportional to the number, $n$, of independently moving objects. Here, however, linear trajectories were more predictable than those random motions. Increased set sizes had smaller 
effects in the present study, but the effects were still substantial.

What, exactly, was the relation between set size and detection rates? For all 48 independent data points in Fig. 4, with 12 different functions relating cumulative hazard values, $B(t)$, to $\log _{2}(n)$, the values of $B(t)$ for set sizes $n=\{2,4,6\}$ averaged $74 \%, 48 \%$, and $32 \%$, respectively, relative to set size 1 (the average correlation between $B(t)$ and $\log _{2}(n)$ at the three times $(600,700$, and $800 \mathrm{~ms})$ was $\left.R^{2}(d f=38)=0.972\right)$. Much more research is necessary, however, to understand and quantify the visual workload imposed by variations in the attentional field.

\section{Mathematical structure of the target detection process}

Quantitative characteristics of the target detection process were described in our previous study (Lappin et al., 2016) by a simple mathematical equation (with minor changes in notation):

$b^{\prime}(t)=V(t) \cdot A(n)^{-1} \cdot C$

where $b^{\prime}(t)$ was the detection hazard rate in bits/s at response time $t ; 0 \leq V(t) \leq 1$, was the linearly increasing proportion of time between the start and completion of visual motion integration (at about 400 and $900 \mathrm{~ms}$, respectively); $A(n)=n$ was the complexity of the attended background, proportional to set size, $n$; and $C \cong 25 \mathrm{bits} / \mathrm{s}$ was a constant parameter representing an inferred channel capacity. Figure 6e shows data that were well described by Eq. (1).

The present results require several changes in this equation. First, the temporal process denoted by the $V(t)$ function was quite different from the linear motion integration found in our previous study. Temporal profiles of the visual signal processes for Color and Direction display changes are described by the adjusted $b^{\prime}(t)$ functions in Fig. 6a, b. Both signal functions in the present study were highly non-monotonic, distinctly different from the linear signal process found previously (Lappin et al., 2016), depicted in Fig. 6e. As theoretical functions are not available, numerical approximations might be obtained by ad hoc curve-fitting, but these would add little to the empirical results. Thus, we use $V(t$, Signal), with Signal $=\{$ Color, Dir $\}$ to stand for the empirical temporal signal processes for the Color- and Direction-changes described by the detection rate functions in Fig. 6a, b. These functions describe the apparent opening and closing of a brief time window of roughly $250 \mathrm{~ms}$ for detecting most of the visual change signals.

Second, Eq. (1), must also be modified to accommodate the Selective response process. We can designate this response process by a function $R(t$, Task $)$, where Task $=\{$ Simple, Select $\}$, and $R(t$, Simple $)=1$; and $R(t$, Select $)$ is a timevarying function defined by the relative detection rates for the Simple and Select tasks: $R(t$, Select $)=b^{\prime}(t, n$, Signal, Select $) / b^{\prime}(t, n$, Signal, Simple). However, because the present computational procedures do not estimate the detection rate functions, $b^{\prime}(t)$, at the same points in time for the Simple and Select tasks, we can use the integrated detection rates to estimate the relative influence of the Selective response task. Temporal integrals, $\int_{0}^{t} R\left(t^{\prime}\right.$, Select $) d t^{\prime}$, are indicated by the relative cumulative values in Fig. 5b, where for $t=\{600,700$, $800,900 \mathrm{~ms}\}, \int_{0}^{t} R\left(t^{\prime}\right.$, Select $) d t^{\prime}=\{0.24,0.45,0.60,0.72\}$ the obtained ratios $B(t$, Select $) / B(t$, Simple).

Third, a significant modification of Eq. (1) involves the attentional field complexity, $A(n)$, based on the effects of set size. Our previous finding that $A(n)=n$ was a special case, as is now obvious from the present data. The pre-target attentional field in the present study was obviously less complex than the number $n$ of independent objects. For set sizes 2, 4, and 6, the attentional field can be approximated by the relative values shown in Fig. $5 \mathrm{c}$, d. Recognizing that the relative performance for $n=1$ vs. $n>1$ changes over time, we might estimate the set size effects by the relative values of cumulative hazard functions at times 600, 700, and $800 \mathrm{~ms}$ in Fig. 4, approximated by the obtained linear relation between $B(t)$ and $\log (n)$. The relative values of $B(t)$ at these times provide estimates of $A(n=\{2$, $4,6\})=\{1.35,2.09$, and 3.08$\}$ relative to $A(1)$. These ad hoc descriptive values are not derived from theory, however, and have unknown generality.

Fourth, the capacity parameter, $C$, appears to have had a slightly lower value here than the value of about $30 \mathrm{bits} / \mathrm{s}$ shown in Fig. 6e for our previous study. Here, the average maxima of the adjusted detection rate functions (adjusted to compensate for the set-size effects) shown in Fig. 6a for the Simple Color task was 20.9 bits/s. We can arbitrarily adopt that value as an estimate for the constant parameter $C$ for the present data. Quantifying the rate of a hypothesized channel capacity certainly requires further research, however.

Thus, the abstract temporal structure of the obtained hazard rates is represented by the following equation,

$b^{\prime}(t)=V(t$, Signal $) \cdot R(t$, Task $) \cdot A(n)^{-1} \cdot C$

where $b^{\prime}(t)$ is the detection hazard rate at time $t$, in bits/s; $V(t$, Signal) specifies the rising and falling visual signal process, 0 $\leq V(t$, Color $) \leq 1, V(t$, Color $)=1$ at the time where $b^{\prime}(t$, Color $)$ is maximum, and $0 \leq V(t$, Dir $)<1 ; R(t$, Task=Simple $)=1$ and $R(t$, Selective $)$ is a time-varying function defined by the relative detection rates for the Simple and Select tasks; $A^{-1}(n) \cong$ $\{0.74,0.48$, and 0.32$\}$ for $n=\{2,4,6\}$; and $C \cong 20.9 \mathrm{bits} / \mathrm{s}$. The two visual signal functions $V(t$, Color $)$ and $V(t$, Dir $)$, are specified empirically by the functions in Fig. 6a, c, and the response function $R(t$, Select $)$ is also defined empirically by proportional detection rates in the Selective task relative to the Simple task, involving the relative values the functions in Fig. $6 \mathrm{~b}$ vs. a and $6 \mathrm{~d}$ vs. c. Currently available evidence and theory are insufficient to specify these visual processes mathematically, so testing Eq. (2) against numerical predictions is not yet 
appropriate. Nevertheless, Eq. (2) describes fundamental temporal characteristics of target detection found in this study.

Specifically, Eq. (2) states that the rate of target detection at any given time, $t$, is given by the numerical product of four mutually independent variables or parameters. Two of these, $V(t$, Signal $)$ and $R(t$, Task), vary with time, while the other two, $A(n)$ and $C$, are constant over time. The time-invariant divisive influence of the attentional field, $A(n)$, is a primary feature of Eq. (2).

The hypotheses expressed in Eq. (2) were tested by the obtained cumulative hazard functions, $B(t)$, for the 16 experimental conditions:

$B(t, n)=C \cdot A(n)^{-1} \int_{0}^{t} V\left(t^{\prime}\right.$, Signal $) \cdot R\left(t^{\prime}\right.$, Task $) d t^{\prime}$.

The comparisons in Fig. 4 of actual and estimated cumulative hazard functions, $B(t)$, at three specific times constitute tests of the hypothesis given by Eq. (3). The hypothesis that effects of the attentional field complexity, $A(n)$, are invariant with time as well as with the Signal and Task variables was tested by the hazard rates, $b^{\prime}(t)$, for three successive 100 -ms intervals. Results in Fig. 5c, d, Table 2, and (less clearly) Table 1 support Eq. (3).

Equations (1) (2), and (3) treat time as an external factor rather than a dependent performance measure. RTs are randomly distributed, but they are treated here as input parameters, as times at which the system is observed. A salient property of Eqs. (1), (2), and (3) is their time-invariance-where the output measure on the left side equals a product of the control parameters and variables on the right side regardless of the response time. The present hazard functions and those of Lappin et al. (2016) support these time-invariant relationships.

Selective influence Empirical support for Eq. (3) rests on the selective influence of independent variables. Selective influence is a symmetry property, where the effects of one independent variable are invariant with changes in performance produced by other variables. Here, effects of the visual signal varied over time and differed for the Color vs. Direction target signals, but the qualitative temporal structure of these signalcontrolled detection rate functions-temporal locations of maxima and minima - were approximately invariant with performance changes due to set size and response task. Similarly, effects of the response task also varied over time and were different for Simple vs. Selective responses, but were also approximately invariant with the effects of the set size and target signals. And the divisive effects of attentional set size depended only on set size, invariant with performance changes over time and over the effects of different visual signals and response tasks. The selective influence of those control variables served to identify the component processes and functional relations described by Eqs. (2) and (3). Factorial experiments are obviously necessary for defining and testing test selective influence (Dzhafarov, 2003; Dzhafarov \& Kujala, 2010, 2012; Jones \& Dzhafarov, 2014; Little et al., 2017; Townsend, 1984).

Less obviously, selective influence depends on the dependent measure. Selective influence was exhibited in these results by symmetries of hazard functions, not by symmetries of relative RTs (see Supplementary Material). Indeed, selective influence on hazard functions usually precludes selective influence on RTs, and conversely. The functional relations described by Eqs. (2) and (3) involve parallel processes with concurrent and independent influence on the detection rates at the same times across conditions. Such symmetrical relations among hazard functions in factorial experiments are associated with parallel processes, while such symmetries among the RTs are associated with serial influence on process rates (see Supplementary Material).

Conclusions Conscious perceptual awareness seems to operate at rates governed by a limiting channel capacity. By measuring hazard functions for factorial combinations of attentional set size, sensory target signals, and response task, this study found that: (1) Hazard rates measure the temporal process of visual target detection. (2) The influence of the attentional field size was approximately constant over time and independent of the effects of the visual target signals and response tasks in this experiment. The set size of the attentional field modified the rates of sensory and decision processes but not the temporal influence functions for these processes. (3) Hazard functions revealed lawful symmetries that were not described by RT measures of process durations.

\section{Supplementary Material}

Computations for testing the temporal constancy of proportional hazard functions for Set Sizes 2, 4, and 6 Computations of $R M S$ deviations in Table 1 involved nine data points - Set Sizes 2, 4, $6 \mathrm{x}$ three 100-ms time intervals beginning at 600, 700 , and $800 \mathrm{~ms}$ - for each of the five participants in each of four target detection conditions. Let these nine hazard rates be $b_{i}^{\prime}\left(t_{j}\right)$ with $i=\{2,4,6\}$ and $j=\{600,700,800\}$. The average hazard rate for each time interval is then $b^{\prime}\left(t_{j}\right)=\Sigma_{i} b_{i}^{\prime}\left(t_{j}\right) / 3$, and the average relative hazard rate for Set Size $i$, relative to the average of the three sizes, is $b_{i}^{\prime}=\left[\Sigma_{j}\left[b_{i}^{\prime}\left(t_{j}\right) / b^{\prime}\left(t_{j}\right)\right]\right] / 3$. We used the same relative set size parameter values, $b_{i}^{\prime}$, for all five participants, based on the average for the five participants (these common set size parameters often yielded better fits to the obtained data than separate parameter estimates for each participant - evidently due to unreliability of the set size parameter estimates for individual participants). Thus, the 45 data points ( 9 x 5 participants) in each of the four target detection conditions were compared with predicted values based 
on 17 parameters -3 time intervals x 5 participants +2 relative set-size parameters.

At each data point, the predicted hazard rate was converted to the number of survivors remaining undetected during that interval. The probability of survival within a given $0.1 s$ interval, $p$, was obtained from the predicted hazard rate by the formula $p=(0.5)^{\left(b^{\prime}(t) / 10\right)}$. If $N$ survivors were not yet detected at the start of a given interval, then $N p$ survivors were expected at the end of the interval.

Differences between observed and predicted numbers of survivors at each data point were evaluated relative to the standard deviation of the binomial distribution, $\sigma_{b}$ $=\sqrt{ }[N p(1-p)]$. Thus, where $O$ and $P$ are the observed and predicted survivors, $(O-P) / \sigma_{b}$ is a standardized measure of deviation between the observed and predicted response frequencies. A measure of total deviation between the observed and predicted values is $R M S=$ $\sqrt{ }\left[\Sigma_{i} \Sigma_{j}\left[\left(O_{i j}-P_{i j}\right)^{2} /\left(N_{i j} p_{i j}\left(1-p_{i j}\right)\right)\right] / d f\right]$. RMS values for each participant were computed by dividing the sums of squared deviations by $d f=5.6$, and by $d f=$ 28 for the total sums of squares for all five participants in each target detection condition. These RMS deviations approximate square roots of averaged squared $z--$ scores, and statistical significance was evaluated by the normal distribution.

Table 2 Computations of $R M S$ deviations in Table 2 were similar to those in Table 1, but in this case were based on nine hazard rates ( 3 set sizes $x 3$ time intervals) for each of the four conditions, not on the data for each individual participant. The pair-wise deviations were based on two sets of nine data points for the pair of conditions, with the same relative hazard rate parameters for both conditions, based on the average values for the two conditions. Thus, predicted hazard rates and hit rates for the pair of conditions were based on eight parameters ( 2 conditions $\mathrm{x} 3$ time intervals +2 relative hazard rates), with $d f=10$; and predictions for the individual conditions were based on five parameters ( 3 time intervals +2 relative hazard rates), with $d f=4$.

Comparing RTs and hazard functions Three principal results described by hazard functions were reexamined with RTs: (a) mutually invariant effects of the independent task variables, (b) momentary rise and fall of detection rates, and (c) temporally constant set size effects. These comparative results show that the temporal processes of target detection were not well described by RTs. The simple effects of set size found here and by Lappin et al. (2016) were not revealed by RT distributions.

First, the results described by hazard functions in Fig. 4 were also described by the average (over five participants) median RTs in each of the 16 experimental conditions (the medians are unaffected by missed detections). These RTs were compared with the values estimated by adding the effects of three independent factors-Color vs. Direction signals, Simple vs. Selective responses, and set size. The obtained and estimated values are shown in Fig. 8. The RT-based estimates of independent effects accounted for $R^{2}(d f=12)=$ $93.1 \%$ of the variance in the observed RTs . In contrast, corresponding estimates for hazard functions accounted for $R^{2}=$ 97.7, 98.1, and 95.8 percent of variance at three successive times (average $R^{2}=97.2$ ), as shown in Fig. 4 . Thus, the percentage of variance not accounted for by independent RT effects was more than twice that for the $B(t)$ measures. The RT data in Fig. 8 also exhibit one cross-over interaction not seen in the hazard functions in Fig. 4. Similar analyses for the 25th, 50th, and 75th percentiles of the cumulative RT distribution function (combined from all five participants) yielded $R^{2}$ values of $96.3,93.9$, and 87.6 percent of the variance in RTs. Thus, the RT results also suggested that the three experimental variables had roughly additive effects, but these effects were less consistent than corresponding effects measured by hazard functions.

Two additional analyses examined the temporal functions of momentary detection rates based on temporal densities of RT response frequencies. The hazard rate measures, $b^{\prime}(t) \approx$ $\Delta B(t) / \Delta t$, in Fig. 3 were estimated by ratios of differences in $B(t)$ relative to differences in RTs for $20 \%$ differences in the survival function. Comparable measures of RT probability densities may be quantified in "fifths per second" (FPS), where $F P S(t)=1 / \Delta t$, and where $\Delta t$ is the difference in RTs for a $20 \%$ reduction in $S(t)$. The $F P S(t)$ and $b^{\prime}(t)$ measures have the same values of RT in the denominator; but the numerator of $F P S(t)$ is simply 1.0 , while the $b^{\prime}(t)$ numerator is a difference between the two $B(t)$ values. Figure 9 gives a graphical comparison of the FPS $(t)$ and $b^{\prime}(t)$ functions, scaled relative to the maximum for Set Size 1 at successive 5-percentile points on the RT distributions for Simple responses to Color signals. Both measures yield rising and falling detection rates, but the shapes and relative values of the four Set Size functions differ. Notably, the FPS $(t)$ functions yield little effect of Set Size at longer RTs, in contrast to the systematic and roughly time-invariant effects revealed by the hazard rate functions.

Figure 9 suggests that $\operatorname{FPS}(t)$ detection-rate measures would not show temporally invariant effects of set sizes. Indeed, that was the case. Relative values of FPS $(t)$ for Set Sizes 2, 4, and 6 were computed for the 600-700, 700-800, and 800-900-ms intervals at each of the four signal $x$ response conditions, to compare with the results shown in Fig. $5 \mathrm{~d}$ for the $b^{\prime}(t)$ measures. Most of the $F P S(t)$ values were not much different from the average for the three set sizes, and the relative values were not consistent over the three time-intervals. Averaged over the four signal $\mathrm{x}$ response conditions and over the three intervals, the relative values for Set Sizes 2, 4, and 6 respectively were $1.03,1.02$, and 0.95 . The important result seen in Fig. $5 \mathrm{~d}$ and Tables 1 and 2 disappeared when described by the RT density function $\operatorname{FPS}(t)$. 


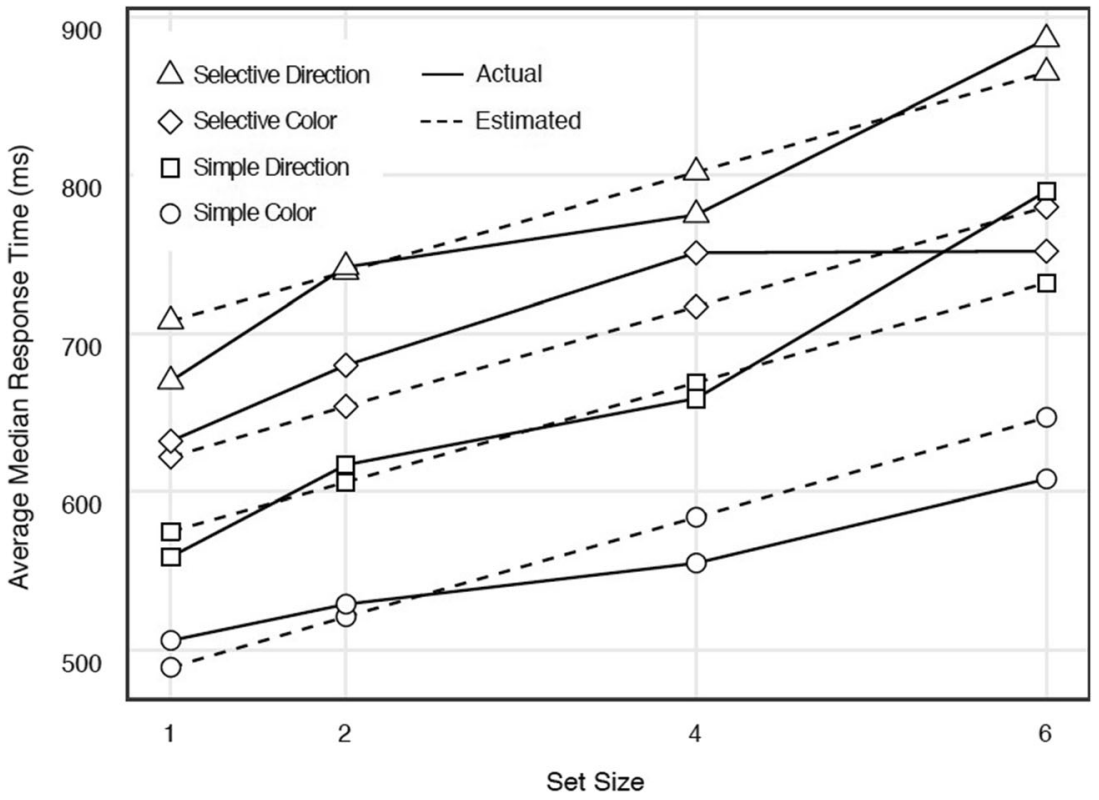

Fig. 8 Estimated and actual average RTs. Actual data, marked by solid lines, are average Median RTs (including undetected targets) for the five participants in each condition. Estimated values, shown by dashed lines, are based on the independent additive effects of the three independent

An analogy may illustrate why measures of hazard functions and measures of RTs provide different function pictures of temporal processes. Imagine that two runners, say F who is faster and $\mathrm{S}$ who runs at half the speed, both complete a 20 mile course in which the first 10 miles are easy but the next 10 miles have hills and obstacles that allow only half the speed. Suppose that $\mathrm{F}$ runs $10 \mathrm{mph}$ for the first half of the course and $5 \mathrm{mph}$ for the second half, while $\mathrm{S}$ runs the first half at $5 \mathrm{mph}$ and the second half at $2.5 \mathrm{mph}$. Analogous to RTs, the runners' relative times at various distances $(\Sigma$ time $/ \Sigma$ distance $)$ measure their relative running speeds: $\mathrm{F}$ finishes in $3 \mathrm{~h}$ and $\mathrm{S}$ in variables - Color vs. Direction signals, Simple vs. Selective responses, and set size. Estimated set size effects are based on the linear regression of RT vs. Set Size $(r=0.991)$. (For log set size, $r=0.973$.)

$6 \mathrm{~h}$, and the same 1/2 ratio holds at each distance. And in this case, their relative speeds (relative distances at given times) are not invariant over time: At 1, 2, and $3 \mathrm{~h}$, F runs 10, 15, and 20 miles, while $\mathrm{S}$ runs 5,10 , and 12.5 miles-yielding performance ratios of $2,1.5$, and 1.6 in relative distance.

Symmetry of the measurement structure reverses, however, when changes in the runners' speeds depend on time rather than distance - e.g., if speeds after the first hour are reduced by changes in weather or visibility. The runners' relative speeds are then measured by their distances at given times: At hours 1, 2, and 3, if F completes 10, 15, and 20 miles, then a

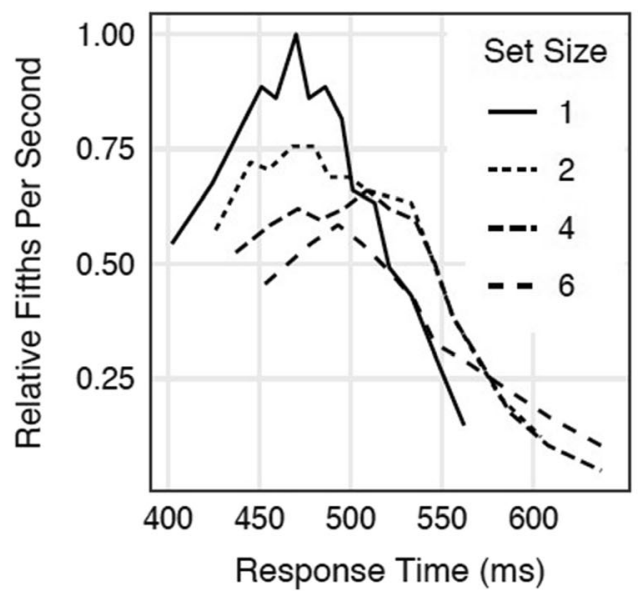

b

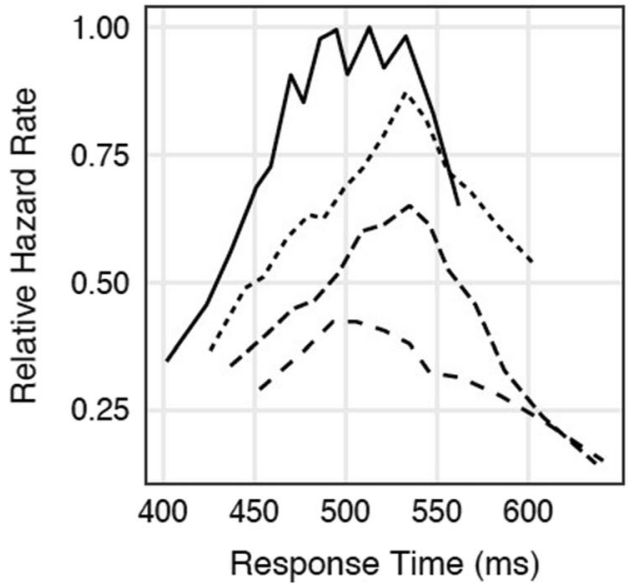

Fig. 9 Temporal processes described by a response frequency densities and $\mathbf{b}$ hazard rates. These functions describe the same set of data, for Simple responses to Color signals 
S completes 5, 7.5, and 10 miles at those times - a constant 2/ 1 ratio in relative distance. Running times as a function of distance, however, the analogs of RTs, are not invariant with distance: At miles 10, 15, and 20, F's times are 1, 2, and $3 \mathrm{~h}$, while S's times at those distances are 3,5 , and $7 \mathrm{~h}$-ratios of $0.33,0.4$, and 0.43 in relative times.

Effects of set size in the present study and in Lappin et al. (2016) were analogous to the latter case: Process rates $(\Delta B(t) /$ $\Delta t$ ) varied with time rather than with progress in detection probability. The symmetrical structure of the present results was measured by cumulative hazard functions rather than by the relative RTs as functions of detection probability. The rationale for measuring RTs vs. hazard functions is empirically testable — by the obtained hazard rate functions.

Random sampling models for serial visual search Simple urn random sampling models serve to describe the qualitative temporal characteristics of serial visual search processes. Consider first the characteristics of random sampling without replacement. Suppose that an urn contains $n$ balls, one of which is red and the remaining $n-1$ are black. Suppose that (a) one ball is taken from the urn at each unit interval in time, where the parameter $t$ counts the number of samples, (b) that the black balls are not replaced after removal from the urn, and (c) that this ball-removal process continues until the red ball is found, stopping at time $t$ when the red ball is removed from the urn. Let $S(t)$ be probability that the red target ball is not found within a series of $t$ samples. Thus, $S(t)$ is given by

$$
\begin{gathered}
S(t)=[(n-1) / n][(n-2) /(n-1)] \ldots[(n-t) /(n-t+1)] \\
=(n-t) / n .
\end{gathered}
$$

Successive samples then reduce the survival function by the probability of target detection in the next sample, $f(t, t+1)$, by

$$
f(t, t+1)=S(t)-S(t+1)=[(n-t) / n]-[(n-t-1) / n]=1 / n .
$$

The hazard function, $h^{\prime}(t)=f(t, t+1) / S(t)$, is then given by

$$
\begin{aligned}
& h^{\prime}(t)=(1 / n) /[(n-t) / n] \\
& h^{\prime}(t)=1 /(n-t)
\end{aligned}
$$

Thus, the hazard rate for random sampling without replacement increases montonically with time $t$ and decreases montonically with set size $n$.

Now consider the temporal process of random sampling with replacement. As before, the model urn contains $n-1$ black balls and one red ball; and the task again involves removing balls at a rate of 1 per time unit, stopping the process as soon as the red target ball is removed. In this task, however, each randomly selected black ball is replaced in the urn immediately after it has been removed. The survival function is then

$S(t)=[(n-1) / n]^{t}$, and the detection probability for the next sample after $t, f(t, t+$ 1) $=S(t)-S(t+1)$, is

$$
\begin{aligned}
f(t, t+1) & =[(n-1) / n]^{t}-[(n-1) / n]^{(t+1)} \\
& =[(n-1) / n]^{t}[1-(n-1) / n],
\end{aligned}
$$

and then the hazard function, $h^{\prime}(t)=f(t, t+1) / S(t)$, is simply

$$
\begin{aligned}
h^{\prime}(t) & =\left[((n-1) / n)^{t}-((n-1) / n)^{(t+1)}\right] /((n-1) / n)^{t} \\
& =1-(n-1) / n \\
h^{\prime}(t) & =1 / n
\end{aligned}
$$

Thus, random sampling with replacement yields temporally constant hazard rates, qualitatively resembling the timeinvariant influence of set size in the present study.

The durations of the serial selection processes described by Eqs. (4) and (5) both increase with set size $n$. Whether or not the set size effects in this study might have involved a serial selection process analogous to that described by Eq. (5), such a serial process cannot account for the temporal structure of the detection rate functions - the temporal locations of maxima and minima - found in this study, which were essentially unaffected by set size.

Acknowledgements This research was supported by a Phase II STTR contract (N00014-15-C-0024) on "Information Salience" from the Office of Naval Research to Discerning Technologies LLC (aka DiscernTek). DiscernTek's CEO, Dr. R. Michael Templeton, provided continuing invaluable assistance throughout both Phase I and II. This project would have been impossible without his supervision and guidance. Technical personnel, facilities, and data collection were provided by Vanderbilt University under a subcontract from DiscernTek. Prof. Gautam Biswas was PI for the Vanderbilt subcontract. We are grateful to Gerald Stanje and Marian Rushdy for their expertise in developing the software that enabled this study. We are grateful to Prof. Daniel Levin for advice in designing the dynamic scenario and for analyzing and reporting data from an associated study (Levin, Biswas, Lappin, Rushdy, \& Seiffert: "Optimistic metacognitive judgments predict poor visual performance in relatively complex visual tasks", (Consciousness and Cognition, 74 (2019), 102781.) We also acknowledge the intellectual contributions of Professors Kazuhiko Kawamura (of Vanderbilt and DiscernTek) and Maithilee Kunda (Vanderbilt) throughout this project. The authors are also grateful to Prof. James Townsend for ongoing discussions of theoretical issues, and to Prof. Michael Wenger and two other anonymous reviewers whose extensive comments on an earlier version prompted corrections and revisions aimed at clarifying the ideas in this manuscript.

\section{References}

Air Combat Command. (2009). Accident Investigation Board Report F-15E, T/N 90-0231, 18 July 2009. Langley AFB, VA: Air Combat Command.

Altieri, N., Stevenson, R.A., Wallace, M.T., \& Wenger, M.J. (2015). Learning to associate auditory and visual stimuli: Behavioral and neural mechanisms. Brain Topography, 28, 479-493. 
Alvarez, G.A., \& Franconeri, S.L. (2007). How many objects can you track? Evidence for a resource limited attentive tracking mechanism. Journal of Vision, 7(13):14, 1-10.

Averbach, E. \& Coriell, A.S. (1961). Short term memory in vision. The Bell System Technical Journal, January 1961, 309-328.

Cavanagh, P. \& Alvarez, G.A. (2005). Tracking multiple targets with multi-focal attention. Trends in cognitive science, 9 (7), 349-354.

Chabris, C. \& Simons, D. (2009). The invisible gorilla. New York: Crown Publishing Group.

Cohen, M.A., Cavanagh, P., Chun, M.M. \& Nakayama, K. (2012). The attentional requirements of consciousness. Trends in Cognitive Science, 16 (8), 411-417.

Coles, M.G.H., Gratton, G., Bashore, T.R., Eriksen, C.W., \& Donchin, E. (1985). A psychophysiological investigation of the continuous flow of human information processing. Journal of Experimental Psychology: Human Perception and Performance, 11, 529-553. https://doi.org/10.1037/0096-1523.11.5.529

Donders, F. C. (1969). On the speed of mental processes (W. Koster, Trans.). In W.G. Koster (Ed.), Attention and performance (Vol. 2, pp. 412-431). Amsterdam: North Holland. (Original work published 1868).

Drew, T., Vo, M.L.-H., \& Wolfe, J.M. (2013). The invisible gorilla strikes again: Sustained inattentional blindness in expert witnesses. Psychological Science, 24 (9), 1848-1853.

Dux, P.E. \& Marois, R. (2009). The attentional blink: A review of data and theory. Attention, Perception, \& Psychophysics, 71(8), 16831700 .

Dzhafarov, E.N. (2003). Selective influence through conditional independence. Psychometrika, 68 (1), 7-25.

Dzhafarov, E.N. \& Kujala, J.V. (2010). The joint distribution criterion and the distance tests for selective probabilistic causality. Frontiers in Quantitative Psychology and Measurement, 1, 151. https://doi.org/ 10.3389/fpsyg.2010.00151.

Dzhafarov, E.N. \& Kujala, J.V. (2012). Selectivity in probabilistic causality: Where psychology runs into quantum physics. Journal of Mathematical Psychology, 56, 54-63.

Eckstein, M.P., Koehler, K., Welbourne, L.E., \& Akbas, E. (2017). Humans, but not deep neural networks, often miss giant targets in scenes, Current Biology, 27, 2827-2832.

Eriksen, B.A. \& Eriksen, C.W. (1974). Effects of noise letters upon the identification of a target letter in a nonsearch task. Perception \& Psychophysics, 16(1), 143-149.

Eriksen, C.W. (1995). The flankers task and response competition: A useful tool for investigating a variety of cognitive problems. Visual Cognition, 2 (2-3), 101-118. https://doi.org/10.1080/ 13506289508401726.

Eriksen, C.W., Coles, M.G.H., Morris, L.R., \& O'Hara, W.P. (1985). An electro-myographic examination of response competition. Bulletin of the Psychonomic Society, 23, 165-168.

Eriksen, C.W. \& Hoffman, J.E. (1973). The extent of processing of noise elements during selective encoding from visual displays. Perception \& Psychophysics, 14 (1), 155-160.

Eriksen, C.W. \& Johnson, H.J. (1964). Storage and decay characteristics of nonattended auditory stimuli. Journal of Experimental Psychology, 68 (1), 28-36.

Eriksen, C.W. \& Lappin, J.S. (1967). Selective attention and very shortterm recognition memory for nonsense forms. Journal of Experimental Psychology, 73(3), 358-364.

Eriksen, C.W. \& Schultz, D.W. (1979). Information processing in visual search: A continuous flow conception and experimental results. Perception \& Psychophysics, 24 (4), 249-263.

Eriksen, C.W. \& St. James, J.D. (1986). Visual attention within and around the field of focal attention: A zoom lens model. Perception \& Psychophysics, 40 (4), 225-240.

Fehd, H.M. \& Seiffert, A.E. (2010). Looking at the center of the targets helps multiple object tracking. Journal of Vision, 10(4):19, 1-13.
Fougnie, D. \& Marois, R. (2006). Distinct capacity limits for attention and working memory: Evidence from attentive tracking and visual working memory paradigms. Psychological Science, 17 (6), 526534.

Fournier, L.R. \& Eriksen, C.W. (1990). Coactivation in the perception of redundant targets. Journal of Experimental Psychology: Human Perception and Performance, 16(3), 538-550.

Franconeri, S.L., Jonathan, S.V., \& Scimeca, J.M. (2010). Tracking multiple objects is limited only by object spacing, not speed, time, or capacity. Psychological Science, 21(7), 920-925.

Garner, W.R. (1962). Uncertainty and structure as psychological concepts. New York: John Wiley.

Gratton, G., Coles, M.G.H., Sirevaag, E.J., Eriksen, C.W. \& Donchin, E. (1988). Pre- and post-stimulus activation of response channels: A psychophysiological analysis. Journal of Experimental Psychology: Human Perception and Performance, 14(3), 331-344.

Houpt, J.W. \& Townsend, J.T. (2012). Statistical measures for workload capacity analysis. Journal of Mathematical Psychology, 56, 341355.

Hyman, R. (1953). Stimulus information as a determinant of reaction time. Journal of Experimental Psychology, 45, 188-196.

Jones, M. \& Dzhafarov, E.N. (2014). Unfalsifiability and mutual translatability of major modeling schemes for choice reaction time. Psychological Review, 121 (1), 1-32.

Kennedy, B.L., Rawding, J., Most, S.B., \& Hoffman, J.E. (2014). Emotion-induced blindness reflects competition at early and late processing stages: An ERP study. Cognitive, affective, and behavioral neuroscience, 14, 1485-1498.

Koenderink, J.J. (2012). Visual awareness. Utrecht, The Netherlands: De Clootcrans Press. (An E-book available at http://www. gestaltrevision.be/pdfs/koenderink/Awareness.pdf.)

Koenderink, J.J. (2018). The way of the eye. Utrecht, The Netherlands: De Clootcrans Press. (An E-book available at http://www. gestaltrevision.be/pdfs/koenderink/Vision.pdf.)

Lappin, J.S. (1967). Attention in the identification of stimuli in complex visual displays. Journal of Experimental psychology, 75 (3), 321328.

Lappin, J.S. \& Ellis, S.H. (1970). The span of apprehension: Form identification as a function of amount of information displayed. Perception \& Psychophysics, 7 (2), 65-72.

Lappin, J.S., Morse, D.L., \& Seiffert, A.E. (2016). The channel capacity of visual awareness divided among multiple moving objects. Attention, Perception, \& Psychophysics, 78, 2469-2493. https:// doi.org/10.3758/s13414-016-1162-z

Levin, D.T., Momen, N., \& Drivdahl, S.B. (2000). Change blindness blindness: The metacognitive error of overestimating changedetection ability. Visual cognition, 7 (1/23), 397-412.

Little, D.R., Altieri, N., Fific, M., \& Yang, C.-T. (2017). Systems factorial technology: A theory driven methodology for the identification of perceptual and cognitive systems. London: Academic Press.

Luce, R.D. (1986). Response times. New York: Oxford University Press.

Luck, S.J. \& Vogel, E. K. (1997). The capacity of visual working memory for features and conjunctions. Nature, 390,279-281.

Luck, S.J. \& Vogel, E. K. (2013). Visual working memory capacity: From psychophysics and neurobiology to individual differences. Trends in Cognitive Sciences, 17, 391-400.

Mack, A. (2003). Inattentional blindness: Looking without seeing. Current Directions in Psychological Science, 12(5), 180-184.

Mack, A. \& Rock, I. (1998). Inattentional blindness. Cambridge, MA: MIT Press.

McClelland, J.L. (1979). On the time relations of mental processes: An examination of systems of processes in cascade. Psychological Review, 86(4), 287-330.

Miller, G.A. (1956). The magical number seven, plus or minus two: Some limits on our capacity for processing information. The Psychological Review, 63 (2), 81-97. 
Miller, J., Riehle, A., \& Requin, J. (1992). Effects of preliminary perceptual output on neuronal activity of the primary motor cortex. Journal of Experimental Psychology: Human Perception and Performance, 18(4), 1121-1138.

Most, S.B., Chun, M.M., Widders, D.M., \& Zald, D.H. (2005). Attentional rubbernecking: Cognitive control and personality in emotion-induced blindness. Psychonomic Bulletin and Review 12, 654-661.

Palmer, E.M., Fencsik, D.E., Flusberg, S.J., Horowitz, T.S., \& Wolfe, J.M. (2011). Signal detection evidence for limited capacity in visual search. Attention, Perception, \& Psychophysics, 73(8), 2413-2424.

Pammer, K., Sabadas, S., \& Lentern, S. (2018). Allocating attention to detect motorcycles: The role of inattentional blindness. Human factors, 60 (1), 5-19.

Pratte, M.S., Young, E.P., Rademaker, R.L., Tong, F. (2017). Accounting for stimulus-specific variation in precision reveals capacity limit in visual working memory. Journal of Experimental Psychology: Human Perception and Performance, 43 (1), 6-17.

Pylyshyn, Z.W. (2004). Some puzzling findings with multiple object tracking: I. Tracking without keeping track of object identities. Visual Cognition, 11, 801-822.

Pylyshyn, Z.W. \& Storm, R.W. (1988). Tracking multiple independent targets: Evidence for a parallel tracking mechanism. Spatial Vision, 3 (3), 179-197.

Shannon, C.E. (1948). The mathematical theory of communication. The Bell System Technical Journal, XXVII (3), 379-423.

Simons, D.J. \& Chabris, C.F. (1999). Gorillas in our midst: sustained inattentional blindness for dynamic events. Perception, 28, 10591074.

Sperling, G. (1960). The information available in brief visual presentations. Psychological Monographs: General and Applied, 74 (11), Whole No. 498, 1-29.

St. James, J.D. \& Eriksen, C.W. (1991). Response competition produces a "fast same effect" in same-different judgments. In G.R. Lockheed \& J.H. Pomerantz (Eds.), The perception of structure (pp. 157-168). Washington, DC: American Psychological Association.

Sternberg, S. (1966). High-speed scanning in human memory. Science, $153,652-654$.

Sternberg, S. (1969). The discovery of processing stages: Extensions of Donders' method. Acta Psychologica. 30. 276-315.

Strayer, D.L., Drews, F.A., \& Johnston, W.A. (2003). Cell phone-induced failures of visual attention during simulated driving. Journal of Experimental Psychology: Applied, 9 (1), 23-32.

Tombu, M. \& Seiffert, A.E. (2008). Attentional costs in multiple-object tracking. Cognition, 108, 1-25

Townsend, J.T. (1971). A note on the identifiability of parallel and serial processes. Perception \& Psychophysics, 10, 161-163.

Townsend, J.T. (1972). Some results concerning the identifiability of parallel and serial processes. British Journal of Mathematical and Statistical Psychology, 25, 168-199.

Townsend, J.T. (1984). Uncovering mental processes with factorial experiments. Journal of Mathematical Psychology, 28, 363-400.

Townsend, J.T. (1990a). The truth and consequences of ordinal differences in statistical distributions: toward a theory of hierarchical inference. Psychological Bulletin, 108, 551-567.

Townsend, J.T. (1990b). Serial vs. parallel processing: Sometimes they look like Tweedledum and Tweedledee, but they can (and should) be distinguished. Psychological Science, 1(1), 46-54.

Townsend, J.T. \& Alteri, N.A. (2012). An accuracy-response time capacity assessment that measures performance against standard parallel predictions. Psychological Review, 119 (3), 500-516.

Townsend, J.T. \& Ashby, F.G. (1978). Methods of modeling capacity in simple processing systems. In J. Castellan \& F. Restle (Eds.)
Cognitive theory, Vol 3, Ch. 8, pp. 199-239. Hillsdale, NJ.: Lawrence Erlbaum Associates.

Townsend, J.T. \& Ashby, F.G. (1983). Stochastic models of elementary psychological processes. Cambridge University Press, Cambridge, U.K.

Townsend, J.T. \& Eidels, A. (2011). Workload capacity spaces: A unified methodology for response time measures of efficiency as workload is varied. Psychonomic Bulletin \& Review, 18, 659-681.

Townsend, J.T. \& Nozawa, G. (1995). Spatio-temporal properties of elementary perception: An investigation of parallel, serial, and coactive theories. Journal of Mathematical Psychology, 39, 321-359.

Townsend, J.T. \& Wenger, M.J. (2004a). The serial-parallel dilemma: A case study in a linkage of theory and method. Psychonomic Bulletin \& Review, 11 (3), 391-418.

Townsend, J.T. \& Wenger, M.J. (2004b). A theory of interactive parallel processing: new capacity measures and predictions for a response time inequality series. Psychological Review, 111, 1003-1035.

Tran, A. \& Hoffman, J.E. (2016). Visual attention is required for multiple object tracking. Journal of experimental psychology: Human perception and performance, 42 (12), 2103-2114.

Treisman, A.M. \& Gelade, G. (1980). A feature-integration theory of attention. Cognitive Psychology, 12, 97-136.

Varakin, D.A., Levin, D.T., \& Fidler, R. (2004). Unseen and unaware: Applications of recent research on failures of visual awareness for human-computer interface design. Human-Computer Interaction, 19, 389-421.

Vogel, E. K., Woodman, G. F., \& Luck, S. J. (2001). Storage of features, conjunctions, and objects in visual working memory. Journal of Experimental Psychology: Human Perception and Performance, 27, 92-114.

Wolfe, J.M., Cave, K.R., \& Franzel, S.L. (1989). Guided search: An alternative to the feature integration model for visual search. Journal of Experimental Psychology: Human Perception and Performance, 15(3), 419-433.

Wolfe, J.M., Soce, A.A., \& Schill, H.M. (2017). How did I miss that? Developing mixed hybrid visual search as a 'model system' for incidental finding errors in radiology. Cognitive Research: Principles and Implications, 2:35, 1-10. https://doi.org/10.1186/ s41235-017-0072-5.

Woodworth, R.S. \& Schlossberg, H. (1954). Experimental psychology (Rev. ed.). New York: Holt, Rinehart \& Winston.

Wundt, W. (1894). Lectures on Human and Animal Psychology, Translated from the Second German Edition, J.E. Creighton \& E.B. Titchener (Eds.). New York: Macmillan.

Young, K. \& Regan, M. (2007). Driver distraction: A review of the literature. In I.J. Faulks, M. Regan, M. Stevenson, J. Brown, A. Porter \& J.D. Irwin (Eds.). Distracted driving (pp. 379-405). Sydney, NSW: Australasian College of Road Safety.

Zhang, W. \& Luck, S.J. (2011). The number and quality of representations in working memory. Psychological Science, 22(11), 14341441.

Open Practices Statement

All of the data collected in this study are available upon request from the first author.

Publisher's note Springer Nature remains neutral with regard to jurisdictional claims in published maps and institutional affiliations. 